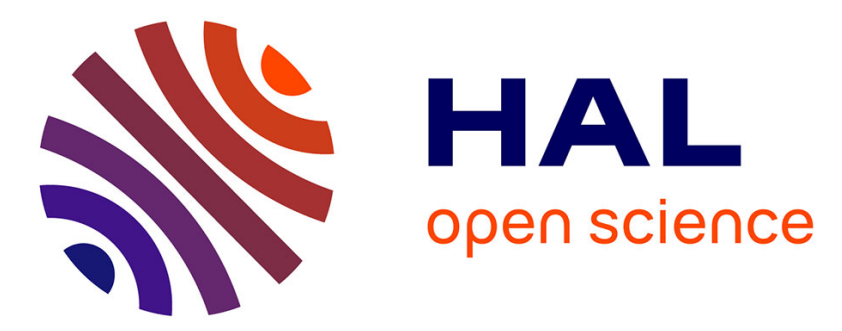

\title{
Solutions of the time-harmonic wave equation in periodic waveguides: asymptotic behaviour and radiation condition
}

Sonia Fliss, Patrick Joly

\section{- To cite this version:}

Sonia Fliss, Patrick Joly. Solutions of the time-harmonic wave equation in periodic waveguides: asymptotic behaviour and radiation condition. Archive for Rational Mechanics and Analysis, 2015, pp.10.1007/s00205-015-0897-3. hal-01086033v2

\section{HAL Id: hal-01086033 \\ https://hal.inria.fr/hal-01086033v2}

Submitted on 17 Jun 2015

HAL is a multi-disciplinary open access archive for the deposit and dissemination of scientific research documents, whether they are published or not. The documents may come from teaching and research institutions in France or abroad, or from public or private research centers.
L'archive ouverte pluridisciplinaire HAL, est destinée au dépôt et à la diffusion de documents scientifiques de niveau recherche, publiés ou non, émanant des établissements d'enseignement et de recherche français ou étrangers, des laboratoires publics ou privés. 
E-mail: sonia.fliss@ensta-paristech.fr 


\title{
Solutions of the time-harmonic wave equation in periodic waveguides : asymptotic behaviour and radiation condition
}

\author{
Sonia Fliss · Patrick Joly
}

the date of receipt and acceptance should be inserted later

\begin{abstract}
In this paper, we give the expression and the asymptotic behaviour of the physical solution of a time harmonic wave equation set in a periodic waveguide. This enables us to define a radiation condition and show wellposedness of the Helmholtz equation set in a periodic waveguide.
\end{abstract}

\section{Introduction}

Periodic media play a major role in applications, in particular in optics for micro and nano-technology $[17,18,23,37]$. From the point of view of applications, one of the main interesting features is the possibility offered by such media of selecting ranges of frequencies for which waves can or cannot propagate. Mathematically, this property is linked to the gap structure of the spectrum of the underlying differential operator appearing in the model. For a complete, mathematically oriented presentation, we refer the reader to $[23,24]$.

This article is a contribution to the theory of time harmonic wave propagation in closed periodic waveguides. More specifically, we are interested in the characterization of outgoing solutions via appropriate radiation conditions, the outgoing solutions being defined a priori thanks to the limiting absorption principle. The construction of these radiation conditions relies on the analysis of the behaviour at infinity of the solution constructed by the limiting absorption process. One particular motivation was to provide preparatory material for the development of the so-called factorization method for inverse scattering problems in locally perturbed periodic waveguides [7] which extensively uses the asymptotic behaviour at infinity of the Green's function associated with the unperturbed periodic waveguide. We also expect that this article is a step toward to the theory of the DtN method developed in $[19,11]$. This method

POems (UMR 7231 CNRS-INRIA-ENSTA), France 
provides transparent boundary conditions for reducing numerical computations to a bounded domain, which has been justified in absorbing media. We expect the extension of these absorbing boundary conditions in the case of non dissipative media, already evoked in [19], to be equivalent to the radiation conditions established in this paper.

It is worth noting that the Russian school [28-30] has extensively contributed to the theory of radiation conditions for wave propagation problems in waveguides using Kondrat'ev's weighted spaces. This approach, which is a priori different from the limiting absorption approach, yields to radiation conditions in waveguides [31] and, more recently, in periodic media [32]. The connection between their approach and ours, which does not appear straighforward to us, would be quite interesting to discover.

The more classical approach to time harmonic solutions of wave propagation problems is the limiting absorption principle; this aims to characterize the physical solution as the limit, when it exists, of the unique solution of finite energy of the corresponding mathematical model with absorption, when this absorption tends to 0 . The corresponding mathematical theory has been extensively developed in the literature in various situations with regard to locally perturbed homogeneous media (see for instance $[9,42,2]$ ) or locally perturbed stratified media (see for instance $[43,8,41,6]$ ). There are much fewer results regarding periodic media. In [12], the authors use Mourre's theory [27,16] to prove the limiting absorption principle for analytically fibered operators whose differential operators with periodic coefficients are a particular case. In [15], the author uses the same theory to deal with the Laplace-Dirichlet operator in a $(\mathrm{n}+1)$-dimensional homogeneous layer with periodically shaped boundary. In [25], a limiting absorption principle for a two-dimensional periodic layer with perturbation is proven. In all these works, the authors are interested mainly in the existence of the limit of the solution with absorption, which amounts to studying the limit of the resolvent of the corresponding periodic differential operator and in which sense this limit holds. Their approach is not constructive and therefore cannot be used to study the asymptotic behavior at infinity of the physical solution and construct corresponding radiation conditions at infinity in order to characterize this solution. That is why we consider a more constructive approach, using the Floquet-Bloch theory, which leads to a semi-analytical expression of the limit, the physical solution, as in [14] for the periodic half-waveguide and in [35] for more general infinite periodic media. In addition, we are able to give a rate of convergence.

The main objective of this work is to use the semi-analytical expression of the solution to deduce, from the analysis of its behavior at infinity, a radiation condition that characterizes this solution, which means that we should be able to show the uniqueness of a solution of the time harmonic wave equation set in a periodic waveguide satisfying this radiation condition. This condition is well-known in the case of a (locally perturbed) homogeneous medium occupy- 
ing the whole space : this is the so called Sommerfeld radiation condition that represents the fact that, at infinity, the solution looks like an outgoing spherical wave which decays as $|x|^{-\frac{d-1}{2}}$ where $d$ is the space dimension. In the case of a homogeneous closed waveguide, that is when the domain of propagation is an infinite cylinder with bounded cross section, the behavior at infinity of the solution is quite different than in the homogeneous whole space : in particular, the solution does not decay any longer at infinity. As a consequence, the radiation condition is of quite different nature and relies on the decomposition of the solution as an infinite sum of evanescent modes and a finite sum of (appropriately chosen) propagative modes : these are the so-called outgoing modes. The situation for a periodic closed waveguide that is considered in this paper is similar in nature to the case of the homogeneous waveguide. However, the notion of outgoing modes is much more delicate (we shall pay a lot of attention to a precise definition and description of such modes) and the analysis relies on quite different mathematical tools (the Floquet-Bloch transform, spectral theory of operators depending analytically on a parameter, complex contour integral techniques etc.).

\section{The model problem}

We consider in this paper the propagation of a time harmonic scalar wave in a perfect periodic waveguide, $\Omega \subset \mathbb{R}^{d+1}$ (typically $d=1$ or 2 ) due to a given source $f$

$$
\begin{cases}-\triangle u-n_{p}^{2} \omega^{2} u=f & \text { in } \Omega \\ \nabla u \cdot \mathbf{n}=0 & \text { on } \partial \Omega\end{cases}
$$

$\mathbf{n}$ being the exterior normal of $\Omega$.

We shall assume that (see Figure 1 for an example)

- The domain of propagation $\Omega$ is a connected open set of $\mathbb{R}^{d+1}$ which is unbounded in one direction, let us set $x_{1}$, bounded in the other ones

$$
\exists \mathbb{S} \text { a compact region of } \mathbb{R}^{d}, \quad \Omega \subset \mathbb{R} \times \mathbb{S},
$$

and periodic with period $L$ in the first direction in the sense that :

$$
\left(x_{1}, x_{s}\right) \in \Omega \quad \Rightarrow \quad\left(x_{1} \pm L, x_{s}\right) \in \Omega .
$$

The boundary $\partial \Omega$ of $\Omega$ is supposed to be smooth enough in order that

$$
\left\{u \in H^{1}(\triangle, \Omega),\left.\nabla u \cdot \mathbf{n}\right|_{\partial \Omega}=0\right\} \subset H^{s}(\Omega) \text { for some } s>\frac{3}{2}
$$

where $H^{1}(\triangle, \Omega)=\left\{u \in H^{1}(\Omega), \triangle u \in L^{2}(\Omega)\right\}$. This is true, for instance, if $\partial \Omega$ is piecewise $C^{1}$ and globally Lipschitz [13]. The unit periodicity cell is defined by

$$
\mathcal{C}=\Omega \cap(-L / 2, L / 2) \times \mathbb{S}
$$


and finally

$$
\Omega=\bigcup_{p \in \mathbb{Z}} \mathcal{C}_{p}, \quad \text { with } \quad \mathcal{C}_{p}=\mathcal{C}+(p L, 0), \forall p \in \mathbb{Z} .
$$

Typically $\Omega$ could be a straight waveguide $(\Omega=\mathbb{S} \times \mathbb{R})$ minus a periodic set of holes (see Figure 1).

- The material properties of the periodic medium, here the refractive index $n_{p}$, is characterized by a $L^{\infty}(\Omega)$ function, with $n_{p} \geq c>0$ which is periodic with the same period than the waveguide

$$
\forall\left(x_{1}, x_{s}\right) \in \Omega, \quad n_{p}\left(x_{1}+L, x_{s}\right)=n_{p}\left(x_{1}, x_{s}\right) .
$$

- The source term represented by the function $f$ is supposed to be $L^{2}$ with a compact support.

Without loss of generality, we suppose in the following that the period $L$ is 1 .

Remark 1 (Extensions) Most of the results of this article can be extended to

- more general symmetric, second order elliptic differential operators with real periodic coefficients (with the same period);

- other boundary conditions as soon as they satisfy the same periodicity properties than the geometry and the coefficients.

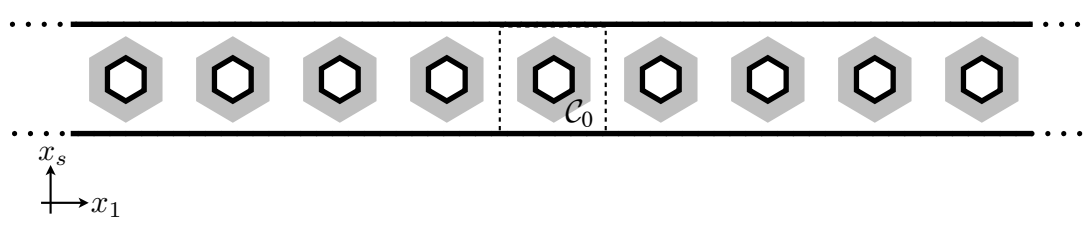

Fig. 1 A periodic domain of propagation : the black lines represents the boundary of $\Omega$ and typically $n_{p}=1$ in the white region, $n_{p}=2$ in the light grey regions.

The principal difficulty of the problem (1) lies in the definition of the notion of the "physical" or "outgoing" solution. Indeed, if the frequency $\omega$ is such that $\omega^{2}$ is in the spectrum of the corresponding periodic differential operator $-n_{p}^{-2} \triangle$ in $\Omega$, waves can propagate in the periodic waveguide, which allows non-vanishing solutions at infinity. In this case, the $H^{1}$ classical framework is not appropriate anymore; solutions cannot exist in this space. On the other hand, in $H_{l o c}^{1}=\left\{u, \quad \forall \varphi \in \mathcal{D}(\mathbb{R}) \quad \varphi\left(x_{1}\right) u\left(x_{1}, x_{s}\right) \in H^{1}(\Omega)\right\}$, one can define several solutions to the original problem. Additional conditions are required to define the "good" physical solution of (1). For example, if the waveguide is straight and the refraction index is constant, the classical separation of variables can be used to show that at infinity any solution is exponentially close to a finite superposition of propagative guided modes. It suffices then to impose 
that this superposition is only made of the outgoing propagative modes on both sides of the support to make the problem well posed; this corresponds to adding a so-called radiation condition. Let us emphasize that the definition of the outgoing modes is explicit in that case thanks to the separations of variables.

In the general periodic case, a classical and natural idea for defining physical solutions is to use the limiting absorption principle. This principle consists in adding some absorption to the original problem (the presence of the damping term $\varepsilon>0$ guaranteeing the well-posedness of this problem in $H^{1}(\Omega)$ )

$$
\begin{cases}-\triangle u_{\varepsilon}-n_{p}^{2}\left(\omega^{2}+\imath \varepsilon\right) u_{\varepsilon}=f & \text { in } \Omega \\ \nabla u_{\varepsilon} \cdot \mathbf{n}=0 & \text { on } \partial \Omega\end{cases}
$$

and in studying the limit, when $\varepsilon$ goes to 0 , of the family of solutions $\left(u_{\varepsilon}\right)_{\varepsilon>0}$. If the limit exists in a certain sense, this limit is defined as the physical or outgoing solution of (1). One says that the limiting absorption principle holds. Applied to the homogeneous waveguide, this approach leads to the outgoing solution as defined in the previous paragraph.

Our main goal in this paper is to characterize the outgoing solution with the help of radiation conditions at infinity, the construction of which relies on the study of the asymptotic behavior of this outgoing solution when $x_{1}$ goes to $\pm \infty$.

The outline of the remainder of the paper is as follows. In Section 3, we introduce useful mathematical tools and we recall and extend (for our purpose) classical results about differential operators with periodic coefficients. In Section 4 , we briefly recall the construction and the semi-analytical expression of the outgoing solution. In Section 5 which is the main section of the paper, we investigate the asymptotic behavior of the outgoing solution when $x_{1}$ tends to $\pm \infty$ and construct the radiation conditions. Finally, in Section 6, we show that this radiation condition ensures the uniqueness of the outgoing solution.

Throughout the remainder of the paper, we will denote for any open set $\mathcal{O} \subset \Omega$

$\forall f, g \in L^{2}(\mathcal{O}), \quad(f, g)_{\mathcal{O}}:=\int_{\mathcal{O}} f \bar{g} d x_{1} d x_{s}, \quad(f, g)_{n_{p}^{2}, \mathcal{O}}:=\int_{\mathcal{O}} f \bar{g} n_{p}^{2} d x_{1} d x_{s}$

and $\|\cdot\|_{\mathcal{O}},\|\cdot\|_{n_{p}^{2}, \mathcal{O}}$ respectively, the corresponding norms. 


\section{Mathematical tools and results on differential operators with} periodic coefficients

3.1 The Floquet Bloch Transform and its properties

We recall the definition and more useful properties of the partial Floquet Bloch Transformation in the $x_{1}$-direction, denoted FBT in the following (see [22] for a more complete and general exposition). Most of the proofs of the forthcoming results are straightforward and left to the reader (see also [22]).

Definition 1 The FBT with period 1 in $\Omega$ is defined by (see [22])

$$
\begin{aligned}
\mathcal{F}: \mathcal{D}(\Omega) & \rightarrow L^{2}(\mathcal{C} \times(-\pi, \pi)) \\
f\left(x_{1}, x_{s}\right) & \rightarrow \hat{f}\left(x_{1}, x_{s} ; k\right):=\frac{1}{\sqrt{2 \pi}} \sum_{p \in \mathbb{Z}} f\left(x_{1}+p, x_{s}\right) e^{-\imath p k},
\end{aligned}
$$

where $\mathcal{D}(\Omega)$ is the set of $C^{\infty}$-functions with compact support (note that the sum in the definition of the FBT is then finite).

Remark 2 The choice of the interval ] $-\pi, \pi[$ for the domain of variation of $k$ is arbitrary. It could be replaced by any interval of lenght $2 \pi$.

The FBT can be extended to $L^{2}$ functions thanks to the following Proposition.

Proposition 1 (Extension of the FBT to $L^{2}(\Omega)$ ) The FBT extends to an isometry between $L^{2}(\Omega)$ and $L^{2}(\mathcal{C} \times(-\pi, \pi))$ :

$$
\forall f, g \in L^{2}(\Omega), \quad \int_{\mathcal{C} \times(-\pi, \pi)} \hat{f} \hat{g} d k d x_{1} d x_{s}=\int_{\Omega} f g d x_{1} d x_{s} .
$$

The second important formula for us is the inversion formula:

Proposition 2 The operator $\mathcal{F}$ is invertible and its inverse is given by $\forall \hat{f} \in L^{2}(\mathcal{C} \times(-\pi, \pi))$, a.e. $\left(x_{1}, x_{s}\right) \in \mathcal{C}, \forall p \in \mathbb{Z}$,

$$
\left(\mathcal{F}^{-1} \hat{f}\right)\left(x_{1}+p, x_{s}\right)=\frac{1}{\sqrt{2 \pi}} \int_{-\pi}^{\pi} \hat{f}\left(x_{1}, x_{s} ; k\right) e^{\imath p k} d k .
$$

In the sequel we shall use the following (obvious) properties of the FBT which make the FBT a privileged tool for the analysis of linear PDEs with periodic coefficients.

Proposition 3 The FBT has the following properties:

1. it commutes with the differential operators, in the sense that

$$
\forall u \in H^{1}(\Omega), \forall k \in[-\pi, \pi], \quad \mathcal{F}\left(\frac{\partial u}{\partial x_{i}}\right)(\cdot ; k)=\frac{\partial}{\partial x_{i}}(\mathcal{F} u(\cdot ; k)) ;
$$


2. it diagonalizes the integer translation operators

$$
\begin{gathered}
\forall\left(x_{1}, x_{s}\right) \in \Omega, \forall p \in \mathbb{Z},\left(\tau_{p} u\right)\left(x_{1}, x_{s}\right)=u\left(x_{1}+p, x_{s}\right) \\
\mathcal{F}\left(\tau_{p} u\right)\left(x_{1}, x_{s} ; k\right)=e^{-\imath p k} \mathcal{F} u\left(x_{1}, x_{s} ; k\right), \quad\left(x_{1}, x_{s} ; k\right) \in \mathcal{C} \times(-\pi, \pi) ;
\end{gathered}
$$

3. it commutes with the multiplication by a periodic function: if $n_{p}$ is a 1periodic $L^{\infty}$-function for all $\left(x_{1}, x_{s} ; k\right) \in \mathcal{C} \times(-\pi, \pi)$

$$
\mathcal{F}\left(n_{p}^{2} u\right)\left(x_{1}, x_{s} ; k\right)=n_{p}^{2}\left(x_{1}, x_{s}\right) \mathcal{F} u\left(x_{1}, x_{s} ; k\right) .
$$

For the sequel, it is essential to know how the Floquet Bloch Transformation acts in Sobolev spaces. We recall these results, a proof of which can be found in $[10,11]$, for instance.

We need first to introduce spaces of functions on the domain $\mathcal{C}$ of so-called $k$ quasi-periodic functions ( $k$ being a parameter in $(-\pi, \pi])$. To do so, we need to introduce the $k$-quasiperiodic extension operator $E_{k} \in \mathcal{L}\left(L^{2}(\mathcal{C}), L_{\text {loc }}^{2}(\Omega)\right)$ defined by (note that $E_{k} f=f$ in $\mathcal{C}$ )

$$
\forall f \in L^{2}(\mathcal{C}), \quad \forall p \in \mathbb{Z}, \forall\left(x_{1}, x_{s}\right) \in \mathcal{C}, \quad E_{k} f\left(x_{1}+p, x_{s}\right)=e^{\imath p k} f\left(x_{1}, x_{s}\right) .
$$

For any $k \in(-\pi, \pi]$ and for any $m \in \mathbb{N}$, let $H_{k}^{m}(\mathcal{C})$ be defined by

$$
H_{k}^{m}(\mathcal{C})=\left\{f \in H^{m}(\mathcal{C}), \quad E_{k} f \in H_{l o c}^{m}(\Omega)\right\},
$$

when $k=0$, the above space will be denoted $H_{\text {per }}^{m}(\mathcal{C})$. One easily shows that $H_{k}^{m}(\mathcal{C})$ is a closed subspace of $H^{m}(\mathcal{C})$. Thus, $H_{k}^{1}(\triangle, \mathcal{C}):=\left\{u \in H_{k}^{1}(\mathcal{C}), \triangle u \in\right.$ $\left.L_{k}^{2}(\mathcal{C})\right\}$ is a closed subspace of $H^{1}(\triangle, \mathcal{C})$, denoted $H_{\text {per }}^{m}(\triangle, \mathcal{C})$ for $k=0$.

Let us give another characterization of $H_{k}^{1}(\mathcal{C})$ and $H_{k}^{1}(\triangle, \mathcal{C})$ which will be more convenient in the sequel.

Proposition 4 For any $k \in(-\pi, \pi]$, a function $u$ belongs to $H_{k}^{1}(\mathcal{C})$ if and only if it belongs to $H^{1}(\mathcal{C})$ and satisfies

$$
\left.u\right|_{x_{1}=1 / 2}=\left.e^{\imath k} u\right|_{x_{1}=-1 / 2}
$$

and it belongs to $H_{k}^{1}(\triangle, \mathcal{C})$ if and only if it belongs to $H^{1}(\triangle, \mathcal{C})$, satisfies (9) and

$$
\left.\partial_{x_{1}} u\right|_{x_{1}=1 / 2}=\left.e^{\imath k} \partial_{x_{1}} u\right|_{x_{1}=-1 / 2}
$$

We can now state the main result for FBT in Sobolev spaces.

Proposition 5 For any $m \in \mathbb{N}, \mathcal{F}$ is an isometric isomorphism from $H^{m}(\Omega)$ into

$\left.H_{Q P}^{m}(\mathcal{C} \times(-\pi, \pi))\right)=\left\{\hat{u} \in L^{2}\left(-\pi, \pi ; H^{m}(\mathcal{C})\right) / \forall k \in(-\pi, \pi), \hat{u}(\cdot ; k) \in H_{k}^{m}(\mathcal{C})\right\}$, equipped with the norm of $L^{2}\left(-\pi, \pi ; H^{m}(\mathcal{C})\right)$ :

$$
\|\hat{u}\|^{2}:=\int_{-\pi}^{\pi}\|\hat{u}(\cdot ; k)\|_{H^{m}(\mathcal{C})}^{2} d k .
$$


Remark 3 We can deduce easily that $\mathcal{F}$ is an isomorphism from $H^{1}(\triangle, \Omega)$ into

$$
\begin{aligned}
H_{\mathrm{QP}}^{1}(\triangle, \mathcal{C} \times(-\pi, \pi))=\left\{\hat{u} \in L^{2}\left(-\pi, \pi ; H^{1}(\triangle, \mathcal{C})\right) /\right. \\
\left.\quad \forall k \in(-\pi, \pi), \hat{u}(\cdot ; k) \in H_{k}^{1}(\triangle, \mathcal{C})\right\},
\end{aligned}
$$

equipped with the norm of $L^{2}\left(-\pi, \pi ; H^{1}(\triangle, \mathcal{C})\right)$ :

$$
\|\hat{u}\|^{2}:=\int_{-\pi}^{\pi}\left[\|\hat{u}(\cdot ; k)\|_{H^{1}(\mathcal{C})}^{2}+\|\triangle \hat{u}(\cdot ; k)\|_{L^{2}(\mathcal{C})}^{2}\right] d k .
$$

\subsection{Analytic families of operators.}

A lot of the technical developments of this paper will be based in complex variable theory and make extensive use of the analytic perturbation theory of operators as described in the reference monography of Kato [21]. In order to make this paper more self-contained, we recall below the various notions of analyticity for families of operators that we will use in the sequel. The only notion that we assume to be known by the reader is the notion of analytic functions with values in a Banach space (see for instance [21], Chapter 3).

Later in the paper, we shall also use various results from the analytic perturbation theory that we shall recall when it will be needed.

In what follows, $\mathcal{O}$ denotes an open domain of the complex plane and $\mathcal{H}$ a given Hilbert space, with scalar product $(\cdot, \cdot)$. We assume that, for any $k \in \mathcal{O}$, we can define an unbounded operator $A(k)$, with domain $D(A(k))$ (including the case where $D(A(k))=\mathcal{H}$ if $A(k)$ is bounded). Doing so, we define a family of operators in $\mathcal{O}$, to which we can attach different notions of analyticity:

Definition 2 The case where the operators $A(k)$ are bounded. In that case the family $A(k)$ is bounded analytic if one of the three equivalent properties is satisfied:

(a) The mapping $k \mapsto A(k)$ is an analytic function from $\mathcal{O}$ with values in $\mathcal{L}(\mathcal{H})$.

(b) For any $f \in \mathcal{H}$, the mapping $k \mapsto A(k) f$ is an analytic function from $\mathcal{O}$ with values in $\mathcal{H}$.

(c) For any $(f, g) \in \mathcal{H}^{2}$, fhe mapping $k \mapsto(A(k) f, g)$ is an analytic function from $\mathcal{O}$ with values in $\mathbb{C}$.

Definition 3 The case where the operators $A(k)$ are unbounded. In this case, we shall refer to two notions of analyticity, namely: 
(a) The family $A(k)$ is analytic of type (A) if the domain of $A(k)$ is independent of $k$, that is $D(A(k))=D$ for any $k$ in $\mathcal{O}$ and, for any $f$ in $D$, the mapping $k \mapsto A(k) f$ is an analytic function from $\mathcal{O}$ with values in $\mathcal{H}$.

(b) The family $A(k)$ is analytic if there exists a family $\widetilde{A}(k)$ of unbounded operators, analytic of type (A), and a family of isomorphism in $\mathcal{H}, S_{k}$, such that $S_{k}$ is bounded analytic (which automatically implies that $S_{k}^{-1}$ is bounded analytic) and

$$
A(k)=S_{k} \widetilde{A}(k) S_{k}^{-1} .
$$

In such a case, if $D$ is the common domain of all the operators $\widetilde{A}(k)$, we have $D(A(k))=S_{k} D$ which means in some sense that the domain of $D(A(k))$ depends analytically on $k$.

The notions of the analyticity of Definition 2 for bounded operators and of analyticity of type (A) of Definition 3 (a) are the ones from Kato's book [21]. The Definition 3 (b) is not given in [21] but it appears to be a particular case of the more general notion of analyticity given on p. 366 of [21] and this notion is also mentioned in Reed and Simon'book [36, Vol. IV, end of p. 20]. What is important for our purpose is that, from the factorization (11), it is quite easy to transfer theorems from perturbation theory of analytic operators of type (A) to analytic operators in the sense of Definition 3 (b).

3.3 The operator $A_{p}$ and its spectral properties

In the following, $L^{2}$ spaces in the variable $\left(x_{1}, x_{s}\right)$ will be naturally equipped with the scalar product associated with the weighted measure $n_{p}^{2} d x_{1} d x_{s}$.

Let us introduce the unbounded operator in $L^{2}(\Omega)$ defined by

$$
A_{p}=-\frac{1}{n_{p}^{2}} \triangle, \quad D\left(A_{p}\right)=\left\{u \in H^{1}(\triangle, \Omega),\left.\nabla u \cdot \mathbf{n}\right|_{\partial \Omega}=0\right\} .
$$

One of the main results of the Floquet-Bloch theory is (see [22] for more details) that the spectrum of $A_{p}, \sigma\left(A_{p}\right)$ is a pure essential spectrum given by

$$
\sigma\left(A_{p}\right)=\bigcup_{k \in]-\pi, \pi]} \sigma\left(A_{p}(k)\right)
$$

where, for all $k \in \mathbb{R}, A_{p}(k)$ is the unbounded operator in $L^{2}(\mathcal{C})$,

$$
A_{p}(k)=-\frac{1}{n_{p}^{2}} \triangle, \quad D\left(A_{p}(k)\right)=\left\{u \in H_{k}^{1}(\triangle, \mathcal{C}),\left.\nabla u \cdot \mathbf{n}\right|_{\partial \Omega \cap \partial \mathcal{C}}=0\right\} .
$$

From the definition of $H_{k}^{1}(\triangle, \mathcal{C})$, it is obvious that $k \mapsto A_{p}(k)$ is $2 \pi$-periodic. For any $k \in \mathbb{R}$, the operator $A_{p}(k)$ is a self-adjoint positive operator with 
compact resolvent so its spectrum is purely discrete. Let us introduce the eigenvalues of the operators $A_{p}(k)$ ordered increasingly,

$$
0 \leq \lambda_{0}(k) \leq \lambda_{1}(k) \leq \ldots \leq \lambda_{n}(k) \leq \ldots, \quad \text { with } \lim _{n \rightarrow+\infty} \lambda_{n}(k)=+\infty
$$

and a corresponding orthonormal basis of eigenvectors $\left(\varphi_{n}(\cdot ; k)\right)_{n \in \mathbb{N}}$. Implicitly, each eigenvalue $\lambda_{n}(k)$ is repeated with its (finite) multiplicity. With this choice, one easily checks that the $\lambda_{n}(k)$ 's have the properties

$$
\forall k \in \mathbb{R}, \quad \lambda_{n}(k+2 \pi)=\lambda_{n}(k) \quad \text { and } \quad \lambda_{n}(k)=\lambda_{n}(-k),
$$

and that the associated eigenvectors can be chosen such that

$$
\forall k \in \mathbb{R}, \quad \varphi_{n}(\cdot ; k+2 \pi)=\varphi_{n}(\cdot ; k) \quad \text { and } \quad \varphi_{n}(\cdot ;-k)=\overline{\varphi_{n}(\cdot ; k)} .
$$

According to [21], the functions $k \mapsto \lambda_{n}(k)$ and $k \mapsto \varphi_{n}(\cdot ; k)$ are continuous and piecewise differentiable functions, with values in $\mathbb{R}$ and $L^{2}(\mathcal{C})$ respectively. These functions lose differentiability only at points $k$ when $\lambda_{n}(k)$ is no longer a simple eigenvalue. There is only a finite number of them and the reason is that these functions are in fact piecewise analytic.

This relies on the perturbation theory of operators [21], and more precisely on the contents of Chapter 7. We first show that the Definition (14) of the operator $A_{p}(k)$ can be extended to $k \in \mathbb{C}$ in such a way that $A_{p}(k)$ depends analytically on $k$ in the sense of the Definition 3 (b). Indeed, we observe that the factorization (11) holds where $S_{k}$ is the isomorphism in $L^{2}(\mathcal{C})$ defined by

$$
S_{k} u(x)=e^{\imath k x} u(x)
$$

(obviously $S_{k}$ is bounded analytic, see Definition 2 ) and $\widetilde{A}_{p}(k)$ the unbounded operator defined by

$\widetilde{A}_{p}(k)=-\frac{1}{n_{p}^{2}}(\nabla+i k)^{2}, \quad D\left(\widetilde{A}_{p}(k)\right)=\left\{u \in H_{p e r}^{1}(\triangle, \mathcal{C}),\left.\nabla u \cdot \mathbf{n}\right|_{\partial \Omega \cap \partial \mathcal{C}}=0\right\}$.

On the other hand, the operators $\widetilde{A}_{p}(k)$ have a fixed domain (that is independent of $k$ ) and depend polynomially on $k$ (and are thus analytic of type $(\mathrm{A})$ ).

Next, a key point is that these operators have a compact resolvent for any $k \in \mathbb{C}$ and are self-adjoint for $k \in \mathbb{R}$. As a consequence, we can refer to the Theorem 3.9, Chapter 7 of [21] and factorization (11) to claim that there exists a sequence of neighborhoods of the real axis $D_{n} \supset \mathbb{R}$, a sequence of analytic functions $\mu_{n}(k): D_{n} \rightarrow \mathbb{C}$ and a sequence of analytic functions $\psi_{n}(\cdot ; k): D_{n} \rightarrow H^{1}(\triangle, \mathcal{C})$ such that for all $k \in \mathbb{R}$

$$
\begin{gathered}
\psi_{n}(\cdot ; k) \in D\left(A_{p}(k)\right), \quad A_{p}(k) \psi_{n}(\cdot ; k)=\mu_{n}(k) \psi_{n}(\cdot ; k) \\
\text { and }\left(\psi_{n}(\cdot ; k), \psi_{m}(\cdot ; k)\right)_{n_{p}^{2}, \mathcal{C}}=\delta_{n, m}
\end{gathered}
$$


As a consequence, for each real $k$, the sequence $\left(\mu_{n}(k)\right)_{n \in \mathbb{N}}$ is simply a rearrangement of the sequence $\left(\lambda_{n}(k)\right)_{n \in \mathbb{N}}$ :

$$
\forall k \in \mathbb{R}, \quad\left\{\mu_{n}(k), n \in \mathbb{N}\right\}=\left\{\lambda_{n}(k), n \in \mathbb{N}\right\} .
$$

Moreover, the eigenfunctions $\varphi_{n}(\cdot ; k)$ can be chosen in such way that

$$
\forall k \in \mathbb{R}, \quad\left\{\psi_{n}(\cdot ; k), n \in \mathbb{N}\right\}=\left\{\varphi_{n}(\cdot ; k), n \in \mathbb{N}\right\} .
$$

We can assume without, any loss of generality, that the domains $D_{n}$ are symmetric neighborhoods of the real axis

$$
k \in D_{n} \quad \Longleftrightarrow \bar{k} \in D_{n} .
$$

We shall call dispersion curves, the curves $k \in \mathbb{R} \mapsto \mu_{n}(k)$. By analyticity arguments, two dispersion curves either coincide along the real axis or meet only a finite number of times inside $[-\pi, \pi]$. Accordingly, for each $n \geq 1$, the interval $[-\pi, \pi]$ can be divided into finite numbers of subintervals inside each of which the function $\lambda_{n}(k)$ (resp. $\varphi_{n}(\cdot ; k)$ ) coincides with some functions $\mu_{p}(k)$ (resp. $\psi_{p}(\cdot ; k)$ ), see Figure 2 for an example which makes precise the definition of piecewise analytic function.

We shall use, in Section 3.4 and 6, another important property of the functions $\mu_{n}(k)$. By analyticity arguments, we know that either $\mu_{n}(k)$ is constant in $[-\pi, \pi]$ or its derivative $\mu_{n}^{\prime}(k)$ vanishes a finite number of times inside $[-\pi, \pi]$. On the other hand, if a function $\mu_{n}(k)$ takes the constant value $\mu$, this means that $\mu$ is an eigenvalue (with infinite multiplicity) of $A_{p}$; in other words, this occurs if and only if the operator $A_{p}$ has a non empty point spectrum, denoted $\sigma_{\mathrm{p}}$. Of course, $\sigma_{\mathrm{p}}$ is necessarily a discrete subset of $\mathbb{R}^{+}$. Furthermore, it is conjectured that this point spectrum is empty and this conjecture has been demonstrated in $[38,40]$ when the transverse space dimension $d=1$. When $d=2$, similar results have been obtained in the (simpler) case of the Schrodinger equation first by [5] for thin tubes, and in [20] for general cylinders. In any case, if $\sigma_{\mathrm{p}} \neq \emptyset$, the values of $\omega$ such that $\omega^{2} \in \sigma_{\mathrm{p}}$ have to be excluded from our analysis. Later, we shall be even led to exclude a largerstill discrete-set of values of $\omega$, denoted $\sigma_{0}$ (see (33)).

Throughout most part of this paper, we shall work with $\left(\mu_{n}(k), \psi_{n}(\cdot ; k)\right)$ instead of $\left(\lambda_{n}(k), \varphi_{n}(\cdot ; k)\right)$. Of course, according to (13), (15) and (19), the spectrum of $A_{p}$ can be characterized by

$$
\sigma\left(A_{p}\right)=\bigcup_{n \in \mathbb{N}} \lambda_{n}([-\pi, \pi])=\bigcup_{n \in \mathbb{N}} \mu_{n}([-\pi, \pi]) .
$$

Let us point out the fact that, contrary to the $\lambda_{n}(k)$ 's, the functions $\mu_{n}(k)$ are not necessarily $2 \pi$-periodic nor even as illustrated in Figure 3 . The counterpart, in terms of the $\mu_{n}(k)$ 's of the evenness of the $\lambda_{n}(k)$ 's is as follows: 


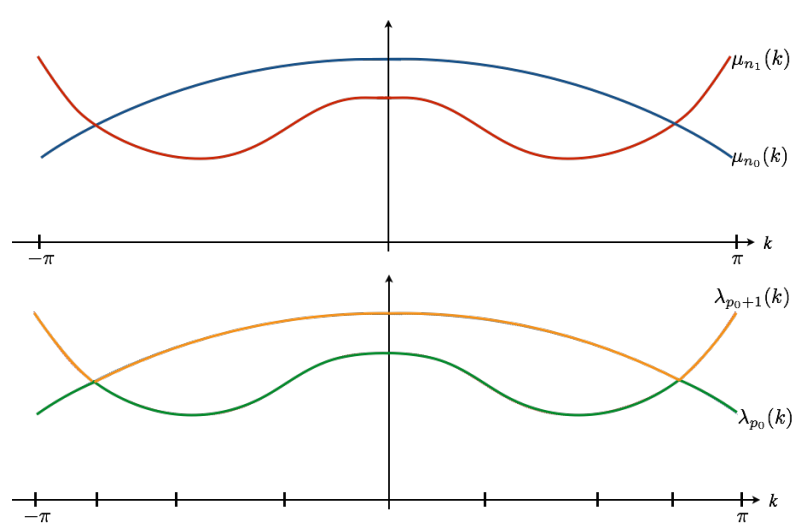

Fig. 2 A picture of two dispersion curves (left) and corresponding $\lambda_{p}(k)$ and $\lambda_{p+1}(k)$ (right)

Lemma 1 For each index $n$, there exists $m$ such that for all $k, \mu_{m}(k)=$ $\mu_{n}(-k)$.

Proof Given $n$, there exists $k$ such that in a neighborhood of $k$, denoted $J$, the multiplicity of $\mu_{n}(-k)$ as an eigenvalue of $A_{p}(-k)$ is constant. As a consequence, by (19), there exists $q \in \mathbb{N}$, depending only on $n$ and $J$, such that for all $\mathrm{k}$ in $\mathrm{J}, \mu_{n}(-k)=\lambda_{q}(-k)$. By the evenness of the $\lambda_{n}(k)^{\prime}$ 's, $\mu_{n}(-k)=\lambda_{q}(k)$ for all $\mathrm{k}$ in J. By similar arguments, there exists $m \in \mathbb{N}$, depending only on $q$ and $J$ such that, for all $\mathrm{k}$ in $\mathrm{J}, \lambda_{q}(k)=\mu_{m}(k)$. Thus $\mu_{n}(-k)$ and $\mu_{m}(k)$ coincide along $J$ and then everywhere by analyticity.

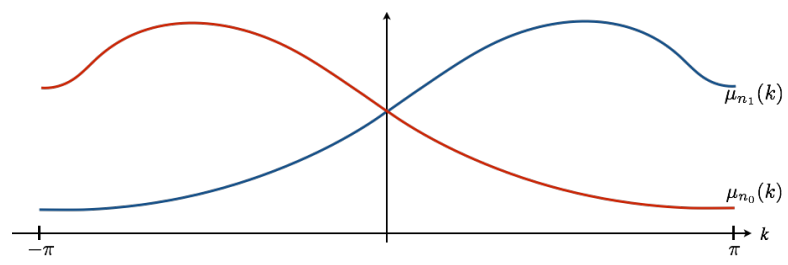

Fig. 3 The dispersion curve $k \mapsto \mu_{n}(k)$ is not necessarily $2 \pi$-periodic nor even

\subsection{The propagating Floquet modes}

In order to define the propagating Floquet modes at a given frequency $\omega$, let us introduce the set of indices $n$ for which, in the $(\xi, \mu)$ plane, the dispersion curve of equation $\mu=\mu_{n}(\xi)$ meets the horizontal line $\mu=\omega^{2}$, namely

$$
\left.I(\omega)=\{n \in \mathbb{N}, \exists \xi \in]-\pi, \pi], \mu_{n}(\xi)=\omega^{2}\right\} .
$$


For each $n \in I(\omega)$, we define the set

$$
\left.\left.\Xi_{n}(\omega)=\{\xi \in]-\pi, \pi\right], \mu_{n}(\xi)=\omega^{2}\right\} .
$$

and the set of propagative wave numbers associated with $\omega$ as (see Figure 4 for an example)

$$
\Xi(\omega)=\bigcup_{n \in I(\omega)} \Xi_{n}(\omega)
$$

In (23), the union is understood in the sense that common elements are repeated in the result, for example

$$
\left\{\xi_{1}, \xi_{2}\right\} \cup\left\{\xi_{1}, \xi_{3}\right\}=\left\{\xi_{1}, \xi_{2}, \xi_{1}, \xi_{3}\right\} .
$$

In this way, each $\xi$ in $\Xi(\omega)$ is associated with a unique dispersion curve $\mu_{n}(\xi)$.

Of course, according to (20), the sets $I(\omega)$ and $\Xi(\omega)$ are empty if and only if $\omega^{2}$ does not belong to the spectrum of $A_{p}$.

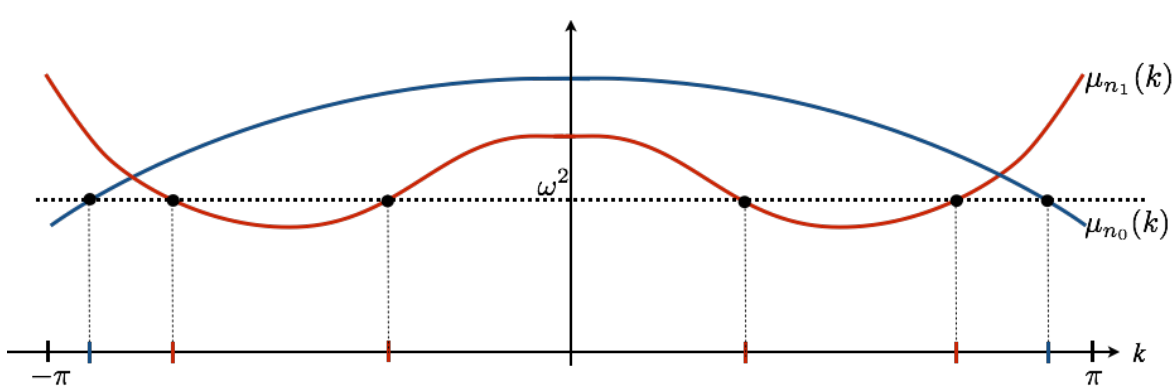

Fig. 4 In this case, $I(\omega)=\left\{n_{0}, n_{1}\right\}, \Xi_{n_{0}}(\omega)$ contains 2 points and $\Xi_{n_{1}}(\omega) 4$ points.

Theorem 1 If $\omega^{2}$ does not belong to the point spectrum of $A_{p}$, the sets $I(\omega)$ and $\Xi_{n}(\omega)$ are finite.

Proof Using the min-max principle and the Weyl estimation (see [36]), it is easy to show that

$$
\forall n, \forall k \in]-\pi, \pi], \quad C_{1} n \leq \lambda_{n}(k) \leq C_{2} n
$$

where $\lambda_{n}(k)$ are the eigenvalues of $A(k)$, ordered increasingly, as defined in Section 3.3 and $C_{1}$ and $C_{2}$ are related to the lower and the upper bounds of $n_{p}$. Using the relation between the $\lambda_{n}(k)$ 's and the $\mu_{n}(k)$ 's, we can show by contradiction that

$$
\lim _{n \rightarrow+\infty} \min _{k \in[-\pi, \pi]} \mu_{n}(k)=+\infty
$$

and thus that $I(\omega)$ is finite. 
Next, let us consider $n \in I(\omega)$. By analyticity arguments, the function $\mu_{n}(k)$ is either constant or meets $\omega^{2}$ only a finite number of times. However, since $\omega^{2}$ does not belong to the point spectrum of $A_{p}$, the first possibility is excluded. Hence, each set $\Xi_{n}(\omega)$ is finite.

On Figure 4 , the sets $\Xi_{n}(\omega)$ are symmetric, however this is not necessarily the case in general, see Figure 5 for an example. Nevertheless, this symmetry property is satisfied by the full set $\Xi(\omega)$.

Theorem 2 The set $\Xi(\omega)$ is symmetric with respect to the origin in the sense that

$$
\xi \in \Xi(\omega) \quad \Rightarrow \quad-\xi \in \Xi(\omega)
$$

Proof This is a straightforward consequence of Lemma 1.

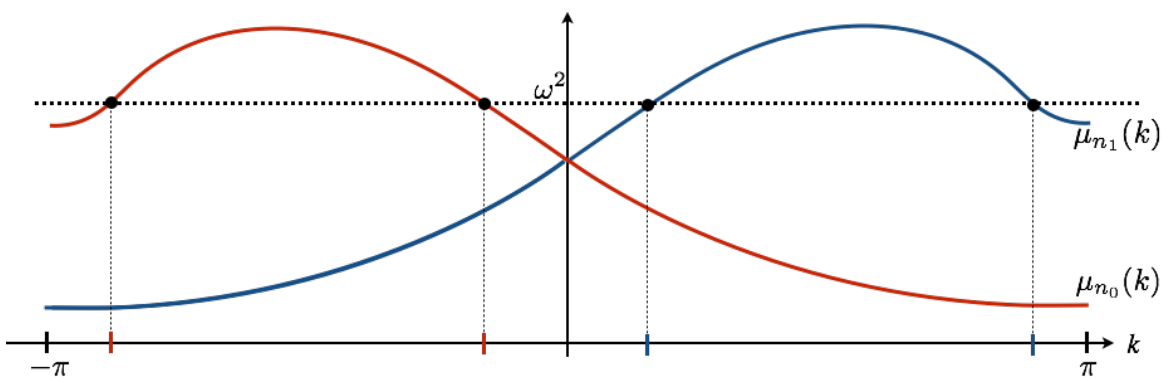

Fig. 5 The sets $\Xi_{n_{0}}(\omega)$ and $\Xi_{n_{1}}(\omega)$ are not symmetric with respect to $k=0$ but $\Xi(\omega)$ is.

For all $n \in I(\omega)$ and $\xi \in \Xi_{n}(\omega)$, let us introduce the corresponding propagating Floquet modes, namely the functions in $H_{l o c}^{1}(\Omega)$ which are the $\xi$-quasiperiodic extensions of the eigenvectors $\psi_{n}(\cdot ; \xi)$, that we still denote with $\psi_{n}$ for simplicity

$$
\psi_{n}(\cdot ; \xi):=E_{\xi} \psi_{n}(\cdot ; \xi) \quad \text { (abuse of notation) }
$$

where the operator $E_{\xi}$ is defined in (8). One easily shows that $\psi_{n}(\cdot ; \xi)$ is an element of the space

$\mathcal{V}(\omega)=\left\{u \in H_{\mathrm{loc}}^{1}(\Omega), \quad-\triangle u-n_{p}^{2} \omega^{2} u=0\right.$ in $\Omega \quad$ and $\quad \nabla u \cdot \mathbf{n}=0$ on $\left.\partial \Omega\right\}$

Furthermore, $\mathcal{V}(\omega)$ is generated by these propagating Floquet modes :

$$
\mathcal{V}(\omega)=\operatorname{span}\left\{\psi_{n}(\cdot ; \xi), \quad \xi \in \Xi_{n}(\omega), n \in I(\omega)\right\} .
$$

On the space $\mathcal{V}=\left\{v \in H_{l o c}^{1}(\Omega), \triangle v \in H_{l o c}^{1} \quad\right.$ and $\quad \nabla v \cdot \mathbf{n}=0$ on $\left.\partial \Omega\right\}$ which contains $\mathcal{V}(\omega)$ for any $\omega$, we can define, setting $\Gamma_{x}=\left\{x_{s},\left(x, x_{s}\right) \in \Omega\right\}$ and using, for $x$ in $\mathbb{R}$, trace theorems on $\Gamma_{x}$ in $H_{\text {loc }}^{s}(\Omega)$ with $s>3 / 2($ see $(2)$ )

$$
\forall x \in \mathbb{R}, \forall v, w \in \mathcal{V}, \quad q(x ; v, w)=\int_{\Gamma_{x}}\left(\frac{\partial v}{d x_{1}} \bar{w}-\frac{\partial \bar{w}}{d x_{1}} v\right) d x_{s} .
$$


Let $x<y$ and $\Omega_{x, y}=\left\{\left(x_{1}, x_{s}\right) \in \Omega, x<x_{1}<y\right\}$, we have by Green's formula

$$
\begin{aligned}
\int_{\Omega_{x, y}}\left[\left(-\triangle-n_{p}^{2} \omega^{2}\right) v \bar{w}-\left(-\triangle-n_{p}^{2} \omega^{2}\right) \bar{w} v\right] & d x_{1} d x_{s} \\
= & q(x ; v, w)-q(y ; v, w) .
\end{aligned}
$$

In particular,

$\forall v, w \in \mathcal{V}(\omega), \forall x \in \mathbb{R}, \quad q(x ; v, w)=q(v, w) \quad$ (independent of $x$ ).

Thus $q(v, w)$ defines a sesquilinear form in $\mathcal{V}(\omega)$, already introduced in [30] where the quadratic form $q(v, v)$ is defined as the energy flux of $v$. One main interest of this sesquilinear form is that it "orthogonalizes" the basis $\left\{\psi_{n}(\cdot ; \xi), \quad \xi \in \Xi_{n}(\omega), n \in I(\omega)\right\}$. This property will be useful to show the uniqueness result of Theorem 8 .

Theorem 3 Let $n, m \in I(\omega), \xi \in \Xi_{n}(\omega), \xi^{\prime} \in \Xi_{m}(\omega)$, then

$$
\begin{array}{ll}
\text { if } m \neq n \text { or } \xi \neq \xi^{\prime} & q\left(\psi_{n}(\cdot ; \xi), \psi_{m}\left(\cdot ; \xi^{\prime}\right)\right)=0 \\
\text { otherwise } & q\left(\psi_{n}(\cdot ; \xi), \psi_{n}(\cdot ; \xi)\right)=\imath \mu_{n}^{\prime}(\xi) .
\end{array}
$$

Proof Writing that for any $n, m \in I(\omega)$ and $\left(\xi, \xi^{\prime}\right) \in \Xi_{n}(\omega) \times \Xi_{m}(\omega)$, $q\left(x ; \psi_{n}(\cdot ; \xi), \psi_{m}\left(\cdot ; \xi^{\prime}\right)\right)$ is independent of $x$, we have, in particular,

$$
\begin{aligned}
\int_{\Gamma_{-1 / 2}}\left(\frac{\partial \psi_{n}(\cdot ; \xi)}{d x_{1}} \overline{\psi_{m}\left(\cdot ; \xi^{\prime}\right)}-\frac{\partial \overline{\psi_{m}\left(\cdot ; \xi^{\prime}\right)}}{d x_{1}} \psi_{n}(\cdot ; \xi)\right) d x_{s} \\
\quad-\int_{\Gamma_{1 / 2}}\left(\frac{\partial \psi_{n}(\cdot ; \xi)}{d x_{1}} \overline{\psi_{m}\left(\cdot ; \xi^{\prime}\right)}-\frac{\partial \overline{\psi_{m}\left(\cdot ; \xi^{\prime}\right)}}{d x_{1}} \psi_{n}(\cdot ; \xi)\right) d x_{s}=0
\end{aligned}
$$

Since $\psi_{n}(\cdot ; \xi)\left(\right.$ resp. $\left.\psi_{m}\left(\cdot ; \xi^{\prime}\right)\right)$ is $\xi\left(\right.$ resp. $\left.\xi^{\prime}\right)$ quasi-periodic, this simply rewrites

$$
\left(e^{\imath\left(\xi-\xi^{\prime}\right)}-1\right) q\left(\psi_{n}(\cdot ; \xi), \psi_{m}\left(\cdot ; \xi^{\prime}\right)\right)=0 .
$$

This gives the result for $\xi \neq \xi^{\prime}$.

We consider now the case $\xi=\xi^{\prime}$. Let $n, m \in I(\omega)$ such that $\mu_{n}(\xi)=\mu_{m}(\xi)=$ $\omega^{2}$. We shall use the derivative of $\psi_{n}(\cdot ; k)$ with respect to $k$, denoted $\partial_{k} \psi_{n}(\cdot ; k)$. The reader will easily recognize that $\partial_{k} \psi_{n}(\cdot ; \xi)$ satisfies

$$
\begin{gathered}
\left(-\triangle-\mu_{n}(\xi) n_{p}^{2}\right) \partial_{k} \psi_{n}(\cdot ; \xi)=\mu_{n}^{\prime}(\xi) n_{p}^{2} \psi_{n}(\cdot ; \xi), \quad \text { in } \Omega \\
\left.\partial_{k} \psi_{n}(\cdot ; \xi)\right|_{\Gamma_{1 / 2}}=\left.e^{\imath \xi} \partial_{k} \psi_{n}(\cdot ; \xi)\right|_{\Gamma_{-1 / 2}}+\left.\imath e^{\imath \xi} \psi_{n}(\cdot ; \xi)\right|_{\Gamma_{-1 / 2}} \\
\left.\partial_{x_{1}}\left[\partial_{k} \psi_{n}(\cdot ; \xi)\right]\right|_{\Gamma_{1 / 2}}=\left.e^{\imath \xi} \partial_{x_{1}}\left[\partial_{k} \psi_{n}(\cdot ; \xi)\right]\right|_{\Gamma_{-1 / 2}}+\left.\imath e^{\imath \xi} \partial_{x_{1}} \psi_{n}(\cdot ; \xi)\right|_{\Gamma_{-1 / 2}}
\end{gathered}
$$


If we use formula (28) with $x=-1 / 2, y=1 / 2, v=\partial_{k} \psi_{n}(\cdot ; \xi) \in \mathcal{V}$ and $w=\psi_{m}(\cdot ; \xi) \in \mathcal{V}$, we obtain

$$
\begin{aligned}
q\left(-1 / 2, \partial_{k} \psi_{n}(\cdot ; \xi), \psi_{m}(\cdot ; \xi)\right)-q & \left(1 / 2, \partial_{k} \psi_{n}(\cdot ; \xi), \psi_{m}(\cdot ; \xi)\right) \\
& =\mu_{n}^{\prime}(\xi) \int_{\mathcal{C}} \psi_{n}(\cdot ; \xi) \overline{\psi_{m}(\cdot ; \xi)} n_{p}^{2} d x_{1} d x_{s}
\end{aligned}
$$

On the other hand, using the boundary conditions (31), (32) and the fact that $\psi_{m}(\cdot ; \xi)$ is $\xi$-quasi-periodic, we obtain after some computations omitted here

$$
\begin{array}{r}
q\left(-1 / 2, \partial_{k} \psi_{n}(\cdot ; \xi), \psi_{m}(\cdot ; \xi)\right)-q\left(1 / 2, \partial_{k} \psi_{n}(\cdot ; \xi), \psi_{m}(\cdot ; \xi)\right) \\
=-\imath q\left(\psi_{n}(\cdot ; \xi), \psi_{m}(\cdot ; \xi)\right)
\end{array}
$$

One then concludes easily by the orthonormality $(18)$ of the $\left(\psi_{n}(\cdot ; k)\right)_{n \in \mathbb{N}}$.

Remark 4 (Physical interpretation) Introducing for all $n \in I(\omega)$ and for all $\xi \in \Xi_{n}(\omega), \omega_{n}(\xi)=\sqrt{\mu_{n}(\xi)}$, one can reinterpret the Floquet mode as the time harmonic wave obeying the dispersion relation

$$
\omega=\omega_{n}(\xi)
$$

and thus define the group velocity by

$$
V_{n}(\xi):=\frac{d \omega_{n}}{d \xi}(\xi)=\frac{1}{2} \mu_{n}(\xi)^{-1 / 2} \mu_{n}^{\prime}(\xi) .
$$

According to the previous Proposition, the sign of the group velocity indicates how the energy propagates in the waveguide.

The propagating Floquet modes can then be divided into those for which $\mu_{n}^{\prime}(\xi)>0$ (they propagate from the left to the right), those for which $\mu_{n}^{\prime}(\xi)<0$ (they propagate from the right to the left) and those for which $\mu_{n}^{\prime}(\xi)=0$.

More precisely, we can decompose the set of propagative wave numbers as

$$
\Xi(\omega)=\Xi^{0}(\omega) \cup \Xi^{+}(\omega) \cup \Xi^{-}(\omega)
$$

where

$$
\begin{gathered}
\Xi^{ \pm}(\omega)=\bigcup_{n \in I(\omega)} \Xi_{n}^{ \pm}(\omega), \quad \Xi_{n}^{ \pm}(\omega)=\left\{\xi \in \Xi_{n}(\omega), \quad \pm \mu_{n}^{\prime}(\xi)>0\right\} \\
\Xi^{0}(\omega)=\bigcup_{n \in I(\omega)} \Xi_{n}^{0}(\omega), \quad \Xi_{n}^{0}(\omega)=\left\{\xi \in \Xi_{n}(\omega), \quad \mu_{n}^{\prime}(\xi)=0\right\}
\end{gathered}
$$

the unions again being defined in the sense of (24).

Theorem 4 The sets $\Xi^{+}(\omega)$ and $\Xi^{-}(\omega)$ are symmetric, one with respect to the other, in the sense that

$$
\xi \in \Xi^{+}(\omega) \quad \Leftrightarrow \quad-\xi \in \Xi^{-}(\omega) .
$$


Proof Let $\xi \in \Xi^{+}(\omega)$. Then there exists $n \in I(\omega)$ such that $\mu_{n}(\xi)=\omega^{2}$ and $\mu_{n}^{\prime}(\xi)>0$. By Lemma 1, there exists $m$ such that for all $k, \mu_{n}(k)=\mu_{m}(-k)$ which implies $-\xi \in \Xi_{m}(\omega)$. Moreover, $\mu_{m}^{\prime}(-\xi)=-\mu_{n}(\xi)<0$ which means that $-\xi \in \Xi_{m}^{-}(\omega)$ and thus to $\Xi^{-}(\omega)$. The converse is proven identically.

We now introduce a set of frequencies $\omega$ for which the limiting absorption principle will not hold

$$
\begin{aligned}
\sigma_{0} & =\left\{\omega \in \mathbb{R}, \exists n, \exists \xi \in(-\pi, \pi], \mu_{n}(\xi)=\omega^{2} \text { and } \mu_{n}^{\prime}(\xi)=0\right\} \\
& \equiv\left\{\omega \in \mathbb{R}, \Xi^{0}(\omega) \neq \emptyset\right\} .
\end{aligned}
$$

Physically, these are the frequencies for which there exists a Floquet mode whose group velocity can vanish. This corresponds, for instance, to the cutoff frequencies for the homogeneous waveguide for which we know that the limiting absorption principle does not hold [26].

Of course the set $\sigma_{0}$ contains the point spectrum $\sigma_{\mathrm{p}}$. However, it is a "quite small" set:

Theorem 5 The set $\sigma_{0}$ is a countable set with at most one point of accumulation $+\infty$.

Proof Let us suppose that there exists a non stationary sequence $\left(\omega_{n}\right)_{n}$ of $\sigma_{0}$, then

$$
\forall n, \exists i_{n}, \exists \xi_{n}, \mu_{i_{n}}\left(\xi_{n}\right)=\omega_{n} \text { and } \mu_{i_{n}}^{\prime}\left(\xi_{n}\right)=0
$$

which converges towards $\omega_{\infty} \in \mathbb{R}^{+}$. The sequence $\left(\omega_{n}\right)_{n}$ is in particular bounded. Moreover, using that (see the proof of Proposition 1)

$$
\lim _{n \rightarrow+\infty} \min _{k \in[-\pi, \pi]} \mu_{n}(k)=+\infty
$$

the set of indices $\left(i_{n}\right)_{n}$ is necessarily finite. Left to extract a subsequence, we can assume that the sequence $\left(i_{n}\right)_{n}$ is constant and equal to $i_{0}$ :

$$
\forall n, \exists \xi_{n}, \mu_{i_{0}}\left(\xi_{n}\right)=\omega_{n} \text { and } \mu_{i_{0}}^{\prime}\left(\xi_{n}\right)=0
$$

The sequence $\left(\omega_{n}\right)_{n}$ is assumed to be non stationary, which implies that $\mu_{i_{0}}^{\prime}\left(\xi_{n}\right)=0$ for a non stationary sequence $\left(\xi_{n}\right)_{n}$. The function $\mu_{i_{0}}$ being analytic implies that $\mu_{i_{0}}$ is a constant, which in turn implies that the sequence $\left(\omega_{n}\right)_{n}$ is stationary, which contradicts the initial assumption.

Remark 5 In Appendix B, we shall show, by explicit calculations, that in a straight homogeneous waveguide, the Floquet modes do coincide with the standard guided modes. 


\section{Limiting absorption principle in the periodic waveguide}

There are some contributions in the study of the limiting absorption principle to periodic waveguides $[25,11,14]$. We remind the reader in this section about the main results and the ideas of the proofs of these works; using the FloquetBloch Transform, we give a semi-analytical expression of the solution of the time-harmonic scalar wave equation (3) with absorption, show that the family of solutions has a limit in a certain sense when the absorption tends to 0 and finally deduce a semi-analytical expression of the physical solution of (1).

Lemma 2 Suppose that $f \in L^{2}(\Omega)$. The solution $u_{\varepsilon}$ of $(3)$ is given by

$$
u_{\varepsilon}=\sum_{n \in \mathbb{N}} u_{\varepsilon}^{n}, \quad \text { in } H^{1}(\triangle, \Omega)
$$

where each $u_{\varepsilon}^{n}$ is given by $\forall\left(x_{1}, x_{s}\right) \in \mathcal{C}, \forall p \in \mathbb{Z}$,

$$
u_{\varepsilon}^{n}\left(x_{1}+p, x_{s}\right)=\frac{1}{\sqrt{2 \pi}} \int_{-\pi}^{\pi} \frac{\mathbb{P}_{n}(f)\left(x_{1}, x_{s} ; k\right)}{\mu_{n}(k)-\left(\omega^{2}+\imath \varepsilon\right)} e^{\imath p k} d k
$$

where

$$
\mathbb{P}_{n}(f)(\cdot ; k)=\left(\hat{f}(\cdot ; k), \psi_{n}(\cdot ; k)\right)_{n_{p}^{2}, \mathcal{C}} \psi_{n}(\cdot ; k)
$$

Proof Applying the Floquet Bloch Transform to Equation (3), and using its properties as detailed in Proposition 3, we see that the FBT of $u_{\varepsilon}$, namely $\hat{u}_{\varepsilon}(\cdot ; k)$, belongs to $D\left(A_{p}(k)\right)$ and satisfies

$$
A_{p}(k) \hat{u}_{\varepsilon}(\cdot ; k)-\left(\omega^{2}+\imath \varepsilon\right) \hat{u}_{\varepsilon}(\cdot ; k)=\frac{1}{n_{p}^{2}} \hat{f}(\cdot ; k) .
$$

After diagonalization of the above equation in the basis $\left(\psi_{n}(\cdot ; k)\right)_{n \in \mathbb{N}}$, we obtain

$$
\hat{u}_{\varepsilon}(\cdot ; k)=\sum_{n \in \mathbb{N}} \frac{\mathbb{P}_{n}(f)(\cdot ; k)}{\mu_{n}(k)-\left(\omega^{2}+\imath \varepsilon\right)}
$$

and finally, by applying the inverse Floquet-Bloch Transform,

$\forall\left(x_{1}, x_{s}\right) \in \mathcal{C}, \forall p \in \mathbb{Z}, \quad u_{\varepsilon}\left(x_{1}+p, x_{s}\right)=\frac{1}{\sqrt{2 \pi}} \int_{-\pi}^{\pi} \sum_{n \in \mathbb{N}} \frac{\mathbb{P}_{n}(f)\left(x_{1}, x_{s} ; k\right)}{\mu_{n}(k)-\left(\omega^{2}+\imath \varepsilon\right)} e^{\imath p k} d k$

To conclude, the only difficulty is to justify the inversion between the integral and the sum. The details use similar arguments to those in Step 1 of Appendix A and are then left to the reader. 
Remark 6 Using the same ideas, we can show that the definition of $\mathbb{P}_{n}(f)$ can be extended if the source term $f \in H^{r}(\Omega)$ for any $r>-s$ with $s>\frac{3}{2}$ because $\psi_{n}(\cdot ; k) \in H^{s}(\mathcal{C})$ (see Assumption (2)). Then the representation of the solution holds in $H^{r+2}(\mathcal{C})$. In particular, we can deduce easily from this result the expression of the Green function $G(\cdot ; y)$ for which formally $f=\delta_{y}$ with $y=\left(y_{1}+q, y_{s}\right),\left(y_{1}, y_{s}\right) \in \mathcal{C}$ and $q \in \mathbb{Z}, \forall\left(x_{1}, x_{s}\right) \in \mathcal{C}, \forall p \in \mathbb{Z}$,

$$
G_{\varepsilon}\left(x_{1}+p, x_{s} ; y_{1}+q, y_{s}\right)=\frac{1}{2 \pi} \sum_{n \in \mathbb{N}} \int_{-\pi}^{\pi} \frac{\psi_{n}\left(x_{1}, x_{s} ; k\right) \overline{\psi_{n}\left(y_{1}, y_{s} ; k\right)}}{\mu_{n}(k)-\left(\omega^{2}+\imath \varepsilon\right)} e^{\imath(p-q) k} d k .
$$

We can now establish the limiting absorption principle which will be proven in Appendix B.

Theorem 6 (Limiting absorption principle) Suppose that $\omega \notin \sigma_{0}$ and $f$ is in $L^{2}(\Omega)$ with a compact support. Then

$$
\forall \eta \in(0,1), \exists C_{\eta}(\omega), \forall p \in \mathbb{Z}, \quad\left\|u_{\varepsilon}-u\right\|_{H^{1}\left(\mathcal{C}_{p}\right)} \leq C_{\eta}(\omega) \varepsilon^{1-\eta}\|f\|_{L^{2}(\Omega)} .
$$

where $u$ is solution of the Helmholtz equation (1) and is given by

$$
u=u_{\text {evan }}+u_{\text {prop }} \text { with } \mid \begin{aligned}
& u_{\text {evan }}=\sum_{n \notin I(\omega)} u^{n} \in H^{1}(\triangle, \Omega) \\
& u_{\text {prop }}=\sum_{n \in I(\omega)}^{n} u^{n} \in H_{\text {loc }}^{1}(\triangle, \Omega)
\end{aligned}
$$

where each $u^{n}$ is given by $\forall\left(x_{1}, x_{s}\right) \in \mathcal{C}, \forall p \in \mathbb{Z}$,

$$
\begin{aligned}
n \notin I(\omega), \quad u^{n}\left(x_{1}+p, x_{s}\right) & =\frac{1}{\sqrt{2 \pi}} \int_{-\pi}^{\pi} \frac{\mathbb{P}_{n}(f)\left(x_{1}, x_{s} ; k\right)}{\mu_{n}(k)-\omega^{2}} e^{\imath p k} d k \\
n \in I(\omega), \quad u^{n}\left(x_{1}+p, x_{s}\right)= & \frac{1}{\sqrt{2 \pi}}\left[p \cdot v \cdot \int_{-\pi}^{\pi} \frac{\mathbb{P}_{n}(f)\left(x_{1}, x_{s} ; k\right)}{\mu_{n}(k)-\omega^{2}} e^{\imath p k} d k\right. \\
& \left.+\imath \pi \sum_{\xi \in \Xi_{n}(\omega)} \frac{\mathbb{P}_{n}(f)\left(x_{1}, x_{s} ; \xi\right)}{\left|\mu_{n}^{\prime}(\xi)\right|} e^{\imath p \xi}\right]
\end{aligned}
$$

and where $\mathbb{P}_{n}(f)$ is defined in (36) and

$$
p . v . \int_{-\pi}^{\pi} \frac{\mathbb{P}_{n}(f)\left(x_{1}, x_{s} ; k\right)}{\mu_{n}(k)-\omega^{2}} e^{\imath p k} d k=\lim _{\delta \rightarrow 0^{+}} \int_{\mathcal{I}_{\omega, n}^{\delta}} \frac{\mathbb{P}_{n}(f)\left(x_{1}, x_{s} ; k\right)}{\mu_{n}(k)-\omega^{2}} e^{\imath p k} d k,
$$

with $\left.\mathcal{I}_{\omega, n}^{\delta}=\right]-\pi, \pi\left[\backslash \bigcup_{\xi \in \Xi_{n}(\omega)}[\xi-\delta, \xi+\delta]\right.$.

Remark 7 When $\omega^{2} \notin \sigma\left(A_{p}\right)$ (that is when $I(\omega)=\emptyset$ ), we show in Appendix A that $u \in H^{1}(\Omega)$ and

$$
\exists C(\omega), \forall p \in \mathbb{Z}, \quad\left\|u_{\varepsilon}-u\right\|_{H^{1}\left(\mathcal{C}_{p}\right)} \leq C(\omega) \varepsilon\|f\|_{L^{2}(\Omega)} .
$$


Remark 8 Suppose that $\omega \notin \sigma_{0}$,

$$
\forall y \in \Omega, \forall p \in \mathbb{Z}, \quad \lim _{\varepsilon \rightarrow 0}\left\|G_{\varepsilon}(\cdot ; y)-G(\cdot ; y)\right\|_{L^{2}\left(\mathcal{C}_{p}\right)}=0
$$

where $G$ is a solution of the Helmholtz Equation (1) with $f=\delta_{y}, y=\left(y_{1}+\right.$ $\left.q, y_{s}\right),\left(y_{1}, y_{s}\right) \in \mathcal{C}$ and $q \in \mathbb{Z}$ and is given by $\forall\left(x_{1}, x_{s}\right) \in \mathcal{C}, \forall p \in \mathbb{Z}$,

$$
\begin{aligned}
& G\left(x_{1}+p, x_{s} ; y_{1}+q, y_{s}\right)= \frac{1}{2 \pi} \sum_{n \notin I(\omega)} \int_{-\pi}^{\pi} \frac{\psi_{n}\left(x_{1}, x_{s} ; k\right) \overline{\psi_{n}\left(y_{1}, y_{s} ; k\right)}}{\mu_{n}(k)-\omega^{2}} e^{\imath(p-q) k} d k \\
&+\frac{1}{2 \pi} \sum_{n \in I(\omega)} {\left[p . v . \int_{-\pi}^{\pi} \frac{\psi_{n}\left(x_{1}, x_{s} ; k\right) \overline{\psi_{n}\left(y_{1}, y_{s} ; k\right)}}{\mu_{n}(k)-\omega^{2}} e^{\imath(p-q) k} d k\right.} \\
&\left.+\imath \pi \sum_{\xi \in \Xi_{n}(\omega)} \frac{\psi_{n}\left(x_{1}, x_{s} ; \xi\right) \overline{\psi_{n}\left(y_{1}, y_{s} ; \xi\right)}}{\left|\mu_{n}^{\prime}(\xi)\right|} e^{\imath(p-q) \xi}\right] .
\end{aligned}
$$

\section{Asymptotic behaviour of the solution when $x_{1}$ tends to $\pm \infty$}

In order to describe the asymptotic behaviour of the solution at $\pm \infty$, we first introduce the notion of functions which are exponentially decreasing at $+\infty$ or $-\infty$ in $H_{\mathrm{loc}}^{1}(\triangle, \Omega)$.

Definition 4 If $m>0$, then $u \in H_{\mathrm{loc}}^{1}(\triangle, \Omega)$ is exponentially decaying in $H_{\text {loc }}^{1}(\triangle, \Omega)$ at $+\infty$ (resp. $\left.-\infty\right)$ if there exist $\alpha>0$ and $C>0$ such that

$$
\left.\|u\|_{H^{1}\left(\triangle, \mathcal{C}_{p}\right)} \leq C e^{-\alpha|p|} \quad \forall p>0 \quad \text { (resp. } \forall p<0\right) .
$$

In this section, we still assume that the source term $f$ has compact support, which implies by the definition of $\mathcal{F}$ (see Definition 1 ), that its Floquet-Bloch transformation $\hat{f}(\cdot ; k)$ can be extended to the complex plane as an analytic function with values in $L^{2}(\mathcal{C})$.

We shall define in this section various domains in the complex plane. The Figure 6 will help the reader to visualize these domains. We suppose now that $\omega \notin \sigma_{0}$ and that $f$ is in $L^{2}(\Omega)$ with a compact support, that as a consequence, Theorem 6 holds. Let us remind the reader that each function $k \mapsto \mu_{n}(k)$ (resp. $k \mapsto \psi_{n}(\cdot ; k)$ ) is analytic in $D_{n}$, a symmetric neighborhood in the complex plane of the real axis (see Section 3.3). Let us introduce

$$
D_{\omega}=\bigcap_{n \in I(\omega)} D_{n}
$$

where, by convention, $D_{\omega}=\emptyset$ when $I(\omega)=\emptyset$ that is to say $\omega^{2} \notin \sigma\left(A_{p}\right)$. When $\omega^{2}$ belongs to $\sigma\left(A_{p}\right), D_{\omega}$ is a symmetric complex neighborhood of the 


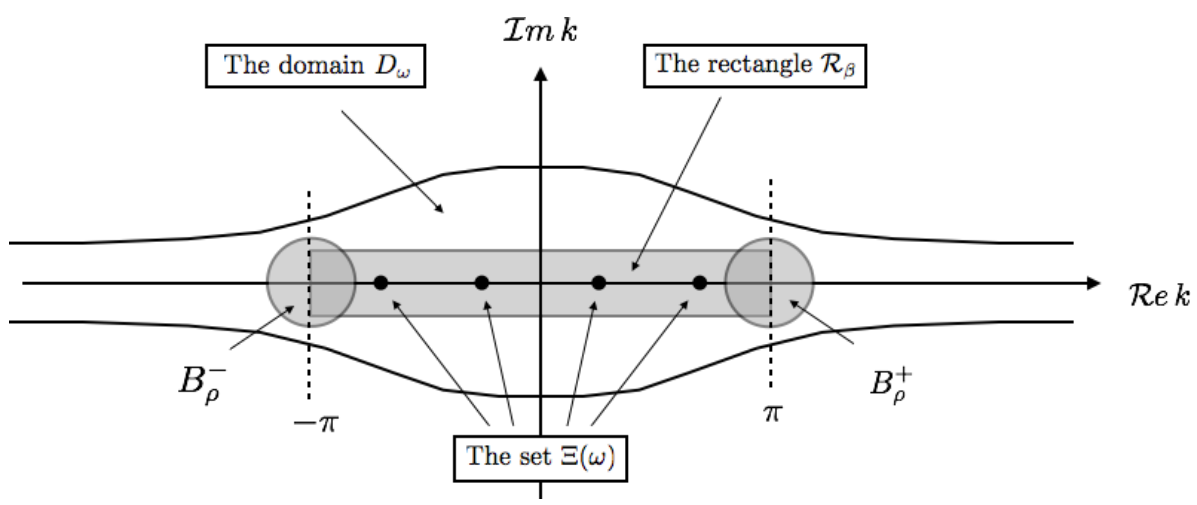

Fig. 6 The various domains in the complex plane

real axis. For each $k \in D_{\omega}$, let us introduce $\mathbb{P}_{\omega}(k)$ and $\mathbb{Q}_{\omega}(k)$ the bounded operators in $L^{2}(\mathcal{C})$ defined by $\mathbb{Q}_{\omega}(k)=\mathbb{I}-\mathbb{P}_{\omega}(k)$ and

$$
\forall g \in L^{2}(\mathcal{C}), \mathbb{P}_{\omega}(k) g=\sum_{n \in I(\omega)}\left(\int_{\mathcal{C}} g \overline{\psi_{n}(\cdot ; \bar{k})} n_{p}^{2} d x\right) \psi_{n}(\cdot ; k) .
$$

Again by convention, $\mathbb{P}_{\omega}(k)=0$ when $\omega^{2} \notin \sigma\left(A_{p}\right)$. These operators obviously satisfy the following properties:

Lemma 3 The operators $\mathbb{P}_{\omega}(k)$ and $\mathbb{Q}_{\omega}(k)$ have the following properties

1. for real $k$, they are orthogonal projectors in $L^{2}(\mathcal{C})$;

2. they are bounded analytic functions of $k$ in $D_{\omega}$.

Proof Since $\mathbb{Q}_{\omega}(k)=\mathbb{I}-\mathbb{P}_{\omega}(k)$, it suffices to give the proof for $\mathbb{P}_{\omega}(k)$.

For the first point, it suffices to notice that for real $k, \bar{k}=k$ so that

$$
\int_{\mathcal{C}} g \overline{\left.\psi_{n}(\cdot ; \bar{k})\right)} n_{p}^{2} d x=\left(g, \psi_{n}(\cdot ; k)\right)_{n_{p}^{2}, \mathcal{C}} .
$$

Thus $\mathbb{P}_{\omega}(k)$ is the orthogonal projector in $L^{2}(\mathcal{C})$ on the space

$$
E_{\omega}=\operatorname{span}\left\{\psi_{n}(\cdot ; k), n \in I(\omega)\right\} .
$$

For the second point, given $(g, h) \in L^{2}(\mathcal{C})^{2}$, we denote

$$
g_{n}(k)=\left(\int_{\mathcal{C}} \bar{g} \psi_{n}(\cdot ; k) n_{p}^{2} d x\right), \quad h_{n}(k)=\left(\int_{\mathcal{C}} \bar{h} \psi_{n}(\cdot ; k) n_{p}^{2} d x\right) .
$$

By analyticity of $k \mapsto \psi_{n}(\cdot ; k) \in L^{2}(\mathcal{C})$, we deduce that both functions $g_{n}(k)$ and $h_{n}(k)$ are analytic functions of $k$ and

$$
\begin{aligned}
\left(\mathbb{P}_{\omega}(k) g, h\right)_{n_{p}^{2}, \mathcal{C}} & =\sum_{n \in I(\omega)}\left(\int_{\mathcal{C}} g \overline{\psi_{n}(\cdot ; \bar{k})} n_{p}^{2} d x\right)\left(\int_{\mathcal{C}} \bar{h} \psi_{n}(\cdot ; k) n_{p}^{2} d x\right) \\
& =\sum_{n \in I(\omega)} \overline{g_{n}(\bar{k})} h_{n}(k)
\end{aligned}
$$


Recalling that if $a(z)$ is analytic in a domain symmetric with respect to the real axis, then $\overline{a(\bar{z})}$ is also analytic, and we deduce that

$$
k \mapsto\left(\mathbb{P}_{\omega}(k) g, h\right)_{n_{p}^{2}, \mathcal{C}}
$$

is analytic in $D_{\omega}$ for any $(g, h) \in L^{2}(\mathcal{C})^{2}$. This allows us to conclude (see Definition 2 (c)).

To study the asymptotic behaviour at infinity of $u$, we shall analyze the sequence $p \mapsto u(\cdot+(p, 0)) \in L^{2}(\mathcal{C})$. We recall (see Theorem 6 ) that $u$ can be written as

$$
u(\cdot+(p, 0))=u_{\text {evan }}(\cdot+(p, 0))+u_{\text {prop }}(\cdot+(p, 0))
$$

where $u_{\text {evan }}$ (the evanescent part of the solution) and $u_{\text {prop }}$ (the propagative part) are given by

$$
\begin{gathered}
u_{\text {evan }}(\cdot+(p, 0))=\frac{1}{\sqrt{2 \pi}} \sum_{n \notin I(\omega)} \int_{-\pi}^{\pi} \frac{\mathbb{P}_{n}(k)(f)(\cdot ; k)}{\mu_{n}(k)-\omega^{2}} e^{\imath p k} d k \\
u_{\text {prop }}(\cdot+(p, 0))=u_{\text {prop }}^{*}(\cdot+(p, 0))+\imath \sqrt{\frac{\pi}{2}} \sum_{n \in I(\omega)} \sum_{\xi \in \Xi_{n}(\omega)} \frac{\mathbb{P}_{n}(f)(\cdot ; \xi)}{\left|\mu_{n}^{\prime}(\xi)\right|} e^{\imath p \xi}
\end{gathered}
$$

where we have defined

$$
u_{\text {prop }}^{*}(\cdot+(p, 0)):=\frac{1}{\sqrt{2 \pi}} \sum_{n \in I(\omega)} p \cdot v \cdot \int_{-\pi}^{\pi} \frac{\mathbb{P}_{n}(f)(\cdot ; k)}{\mu_{n}(k)-\omega^{2}} e^{\imath p k} d k .
$$

We begin with the evanescent term $u_{\text {evan }}(\cdot+(p, 0))$. From Formula (43) and Formula (4), we first reinterpret $u_{\text {evan }}$ as the inverse FBT of

$$
\hat{u}_{\text {evan }}(\cdot ; k)=\sum_{n \notin I(\omega)} \frac{\mathbb{P}_{n}(f)\left(x_{1}, x_{s} ; k\right)}{\mu_{n}(k)-\omega^{2}} \in \operatorname{Im} \mathbb{Q}_{\omega}(k) .
$$

Next we notice that $\hat{u}_{\text {evan }}$ satisfies the equation

$$
\left(A_{p}(k) \mathbb{Q}_{\omega}(k)-\omega^{2}\right) \hat{u}_{\text {evan }}(\cdot ; k)=\mathbb{Q}_{\omega}(k) \hat{f}(\cdot ; k) .
$$

Moreover, it is easy to see that the spectrum of the operator $A_{p}(k) \mathbb{Q}_{\omega}(k)$ is purely discrete and

$$
\sigma\left(A_{p}(k) \mathbb{Q}_{\omega}(k)\right)=\{0\} \bigcup\left\{\mu_{n}(k), n \notin I(\omega)\right\} .
$$

We have then

$$
\bigcup_{k \in]-\pi, \pi]} \sigma\left(A_{p}(k) \mathbb{Q}_{\omega}(k)\right)=\{0\} \bigcup \sigma(A) \backslash J
$$


where $\mathrm{J}$ is an open interval given by

$$
J=\bigcup_{n \in I(\omega)} \mu_{n}(]-\pi, \pi[) .
$$

From Lemma 3, we deduce that $k \mapsto A_{p}(k) \mathbb{Q}_{\omega}(k)$ is analytic in $D_{\omega}$, in the sense of Definition 3 (b). Indeed, setting $\tilde{\mathbb{Q}}_{\omega}(k)=S_{k}^{-1} \mathbb{Q}_{\omega}(k) S_{k}$ which is bounded analytic as product of bounded analytic operators, we can write

$$
A_{p}(k) \mathbb{Q}_{\omega}(k)=S_{k} \tilde{A}_{p}(k) \tilde{\mathbb{Q}}_{\omega}(k) S_{k}^{-1}
$$

where $\tilde{A}_{p}(k) \tilde{\mathbb{Q}}_{\omega}(k)$ is analytic of type $A$ as a product of an analytic operator of type A with a bounded analytic one. Moreover, by definition of $I(\omega), \omega^{2}$ belongs to $J$ and thus, by (46), does not belong to the spectrum of $A_{p}(k) \mathbb{Q}_{\omega}(k)$. Therefore, for any real $k, A_{p}(k) \mathbb{Q}_{\omega}(k)-\omega^{2}$ is invertible from $H_{k}^{1}(\triangle, \Omega)$ into $L^{2}(\mathcal{C})$ with bounded inverse

$$
\forall k \in \mathbb{R}, \quad\left(A_{p}(k) \mathbb{Q}_{\omega}(k)-\omega^{2}\right)^{-1} \in \mathcal{L}\left(L^{2}(\mathcal{C})\right) .
$$

Using [21, Chapter VII, Theorem 1.3] for each $k_{0} \in \mathbb{R}$, there exists a neighborhood $\mathcal{V}\left(k_{0}\right)$ of $k_{0}$, of the form $\left|k-k_{0}\right|<\rho\left(k_{0}\right)$ in which $A_{p}(k) \mathbb{Q}_{\omega}(k)-\omega^{2}$ is invertible from $H_{k}^{1}(\triangle, \Omega)$ into $L^{2}(\mathcal{C})$ and such that the function

$$
k \mapsto\left(A_{p}(k) \mathbb{Q}_{\omega}(k)-\omega^{2}\right)^{-1}
$$

is bounded analytic in $\mathcal{V}\left(k_{0}\right)$. By compactness arguments, using a locally finite covering of the real axis by such neighborhoods, we deduce that there exists a neighborhood of the real axis such that the function

$$
k \mapsto\left(A_{p}(k) \mathbb{Q}_{\omega}(k)-\omega^{2}\right)^{-1}
$$

is well defined and bounded analytic. Without loss of generality, up to redefining $D_{\omega}$ as the intersection between this domain and $D_{\omega}$, we can assume that this domain contains $D_{\omega}$. Next, we observe that, as soon as $\beta>0$ is small enough, we have the inclusion

$$
\mathcal{R}_{\beta} \subset D_{\omega}
$$

where $\mathcal{R}_{\beta}$ is the closed rectangle (see Figure 6 )

$$
\mathcal{R}_{\beta}=\{k \in \mathbb{C}, \operatorname{Re}(k) \in[-\pi, \pi],|\operatorname{Im}(k)| \leq \beta\} .
$$

Since $k \mapsto \hat{f}(\cdot ; k)$ is an analytic function in $\mathbb{C}$ with values in $L^{2}(\mathcal{C})$, the function $\hat{u}_{\text {evan }}(\cdot ; k)$ can then be extended analytically to $k \in D_{\omega}$ via the expression

$$
\forall k \in D_{\omega}, \quad \hat{u}_{\text {evan }}(\cdot ; k)=\left(A_{p}(k) \mathbb{Q}_{\omega}(k)-\omega^{2}\right)^{-1} \mathbb{Q}_{\omega}(k) \hat{f}(\cdot ; k) .
$$

Therefore, (43) can be rewritten as

$$
u_{\text {evan }}(\cdot+(p, 0))=\frac{1}{\sqrt{2 \pi}} \int_{-\pi}^{\pi}\left(A_{p}(k) \mathbb{Q}_{\omega}(k)-\omega^{2}\right)^{-1} \mathbb{Q}_{\omega}(k) \hat{f}(\cdot ; k) e^{+\imath p k} d k,
$$

which we shall use to prove 
Lemma 4 There exist two functions $w_{\text {evan }}^{ \pm} \in H_{\text {loc }}^{1}(\triangle, \Omega)$ such that

$$
\begin{aligned}
u_{\text {evan }}(\cdot+(p, 0)) & =w_{\text {evan }}^{+}(\cdot+(p, 0)) \\
& -\frac{1}{\sqrt{2 \pi}} \int_{\pi}^{\pi+\imath \beta}\left(A_{p}(k) \mathbb{Q}_{\omega}(k)-\omega^{2}\right)^{-1} \mathbb{Q}_{\omega}(k) \hat{f}(\cdot ; k) e^{\imath p k} d k \\
& +\frac{1}{\sqrt{2 \pi}} \int_{-\pi}^{-\pi+\imath \beta}\left(A_{p}(k) \mathbb{Q}_{\omega}(k)-\omega^{2}\right)^{-1} \mathbb{Q}_{\omega}(k) \hat{f}(\cdot ; k) e^{\imath p k} d k \\
u_{\text {evan }}(\cdot+(p, 0)) & =w_{\text {evan }}^{-}(\cdot+(p, 0)) \\
& +\frac{1}{\sqrt{2 \pi}} \int_{\pi-\imath \beta}^{\pi}\left(A_{p}(k) \mathbb{Q}_{\omega}(k)-\omega^{2}\right)^{-1} \mathbb{Q}_{\omega}(k) \hat{f}(\cdot ; k) e^{\imath p k} d k \\
& -\frac{1}{\sqrt{2 \pi}} \int_{-\pi-\imath \beta}\left(A_{p}(k) \mathbb{Q}_{\omega}(k)-\omega^{2}\right)^{-1} \mathbb{Q}_{\omega}(k) \hat{f}(\cdot ; k) e^{\imath p k} d k
\end{aligned}
$$

where $w_{\text {evan }}^{+}\left(\right.$resp. $\left.w_{\text {evan }}^{-}\right)$is exponentially decaying at $+\infty($ resp. $-\infty)$.

Proof

Let us give the proof of the first formula in the lemma. To do so, let us introduce a contour in the complex plane, included in $D_{\omega}$ :

$$
\Gamma_{\omega, \beta}:=\partial \mathcal{R}_{\beta}=[-\pi, \pi] \cup S^{\beta} \cup S_{-}^{\beta} \cup S_{+}^{\beta}
$$

where

$$
\begin{array}{ll}
\left.S^{\beta}=\right]-\pi, \pi[+\imath \beta & \text { horizontal segment } \\
\left.S_{ \pm}^{\beta}= \pm \pi+\imath\right] 0, \beta[ & \text { vertical segment }
\end{array}
$$

and where $\Gamma_{\omega, \beta}$ is oriented according to the Figure 7.

Using the analyticity of $\left(A_{p}(k) \mathbb{Q}_{\omega}(k)-\omega^{2}\right)^{-1} \mathbb{Q}_{\omega}(k) \hat{f}(\cdot ; k) e^{\imath p k}$, we can apply

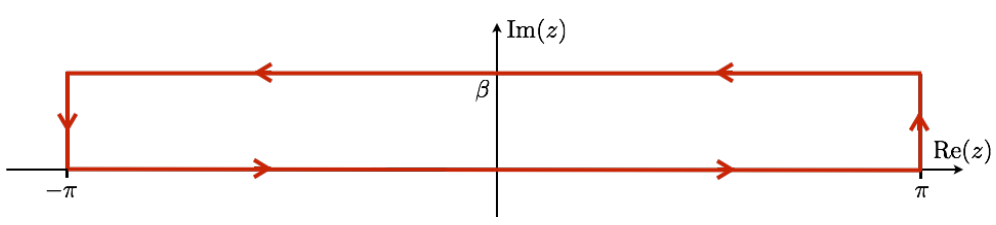

Fig. 7 Oriented contour $\Gamma_{\omega, \beta}$ 
Cauchy's formula to transform the right hand side of (47) as

$$
\begin{aligned}
u_{\text {evan }}(\cdot+(p, 0)) & =w_{\text {evan }}^{+}(\cdot+(p, 0)) \\
& -\frac{1}{\sqrt{2 \pi}} \sum_{ \pm} \int_{S_{ \pm}^{\beta}}\left(A_{p}(k) \mathbb{Q}_{\omega}(k)-\omega^{2}\right)^{-1} \mathbb{Q}_{\omega}(k) \hat{f}(\cdot ; k) e^{\imath p k} d k
\end{aligned}
$$

where, parametrizing $S^{\beta}$ by $k+i \beta, k \in[-\pi, \pi]$,

$$
w_{\text {evan }}^{+}(\cdot+(p, 0))=e^{-p \beta} \frac{1}{\sqrt{2 \pi}} \int_{-\pi}^{\pi} \mathbb{R}_{\omega}^{\text {evan }}(k+\imath \beta) \hat{f}(\cdot ; k+\imath \beta) e^{\imath p k} d k,
$$

where we have set

$$
\mathbb{R}_{\omega}^{\text {evan }}(z):=\left(A_{p}(z) \mathbb{Q}_{\omega}(z)-\omega^{2}\right)^{-1} \mathbb{Q}_{\omega}(z), \quad \forall z \in D_{\omega} .
$$

Setting $C_{\beta}^{\text {evan }}:=\sup _{z \in S^{\beta}}\left\|\mathbb{R}_{\omega}^{\text {evan }}(z)\right\|_{\mathcal{L}\left(L^{2}(\mathcal{C}), H^{1}(\triangle, \mathcal{C})\right)}$, we thus have

$$
\left\|w_{\text {evan }}^{+}\right\|_{H^{1}\left(\triangle, \mathcal{C}_{p}\right)} \leq C_{\beta}^{\text {evan }} e^{-p \beta} \sup _{z \in S^{\beta}}\|\hat{f}(\cdot ; z)\|_{L^{2}(\mathcal{C})} .
$$

The proof for the other formula is obtained by changing the contour $\Gamma_{\omega, \beta}$ by its symmetric with respect to the real axis.

We are now going to focus on $u_{\text {prop }}^{*}$ defined by (45).

Lemma 5 There exist two functions $w_{\text {prop }}^{ \pm} \in H_{\text {loc }}^{1}(\triangle, \Omega)$ such that

$$
\begin{aligned}
u_{\text {prop }}^{*}(\cdot+(p, 0)) & =\imath \sqrt{\frac{\pi}{2}} \sum_{n \in I(\omega)} \sum_{\xi \in \Xi_{n}(\omega)} \frac{\mathbb{P}_{n}(f)(\cdot ; \xi)}{\mu_{n}^{\prime}(\xi)} e^{\imath p \xi}+w_{\text {prop }}^{+}(\cdot+(p, 0)) \\
& -\frac{1}{\sqrt{2 \pi}} \int_{\pi}^{\pi+\imath \beta}\left(A_{p}(k)-\omega^{2}\right)^{-1} \mathbb{P}_{\omega}(k) \hat{f}(\cdot ; k) e^{\imath p k} d k \\
& +\frac{1}{\sqrt{2 \pi}} \int_{-\pi}^{-\pi+\imath \beta}\left(A_{p}(k)-\omega^{2}\right)^{-1} \mathbb{P}_{\omega}(k) \hat{f}(\cdot ; k) e^{\imath p k} d k \\
u_{p r o p}^{*}(\cdot+(p, 0))= & -\imath \sqrt{\frac{\pi}{2}} \sum_{n \in I(\omega)} \sum_{\xi \in \Xi_{n}(\omega)} \frac{\mathbb{P}_{n}(f)(\cdot ; \xi)}{\mu_{n}^{\prime}(\xi)} e^{\imath p \xi}+w_{p r o p}^{-}(\cdot+(p, 0)) \\
& +\frac{1}{\sqrt{2 \pi}} \int_{\pi-\imath \beta}^{\pi}\left(A_{p}(k)-\omega^{2}\right)^{-1} \mathbb{P}_{\omega}(k) \hat{f}(\cdot ; k) e^{\imath p k} d k \\
& -\frac{1}{\sqrt{2 \pi}} \int_{-\pi-\imath \beta}\left(A_{p}(k)-\omega^{2}\right)^{-1} \mathbb{P}_{\omega}(k) \hat{f}(\cdot ; k) e^{\imath p k} d k
\end{aligned}
$$

where $w_{\text {prop }}^{+}\left(\right.$resp. $\left.w_{\text {prop }}^{-}\right)$is exponentially decaying at $+\infty$ (resp. $\left.-\infty\right)$. 
Proof By definition of the principal value, $\forall\left(x_{1}, x_{s}\right) \in \mathcal{C}, \forall p \in \mathbb{Z}$

$$
\sqrt{2 \pi} u_{\text {prop }}^{*}(\cdot+(p, 0))=\lim _{\delta \rightarrow 0} \sum_{n \in I(\omega)} \int_{\mathcal{I}_{\omega}^{\delta}} \frac{\mathbb{P}_{n}(f)(\cdot ; k)}{\mu_{n}(k)-\omega^{2}} e^{\imath p k} d k
$$

where

$$
\left.\mathcal{I}_{\omega}^{\delta}=[-\pi, \pi] \backslash \bigcup_{\xi \in \Xi(\omega)}\right] \xi-\delta, \xi+\delta[.
$$

When $k$ belongs to $\mathcal{I}_{\omega}^{\delta}, \omega^{2}$ belongs to the resolvent set of $A(k)$ and we can write

$$
\forall k \in \mathcal{I}_{\omega}^{\delta}, \quad \sum_{n \in I(\omega)} \frac{\mathbb{P}_{n}(f)(\cdot ; k)}{\mu_{n}(k)-\omega^{2}}=\left(A_{p}(k)-\omega^{2}\right)^{-1} \mathbb{P}_{\omega}(k) \hat{f}(\cdot ; k) .
$$

Looking at the left hand side of (49) and using the analyticity property of $k \mapsto \mu_{n}(k)$ and $k \mapsto \psi_{n}(\cdot ; k)$, we see that the function

$$
k \mapsto \hat{F}_{\omega}(\cdot ; k):=\left(A_{p}(k)-\omega^{2}\right)^{-1} \mathbb{P}_{\omega}(k) \hat{f}(\cdot ; k)
$$

can be extended analytically in $D_{\omega} \backslash \Xi(\omega)$.

Now we want to prove the first formula of the lemma. For this, given $0<\delta<\beta$, let us introduce a contour in the complex plane,

$$
\Gamma_{\omega, \delta, \beta}=\mathcal{I}_{\omega}^{\delta} \cup S^{\beta} \cup S_{-}^{\beta} \cup S_{+}^{\beta} \cup \bigcup_{\xi \in \Xi(\omega)} \gamma_{\delta}(\xi)
$$

where $S^{\beta}$ and $S_{ \pm}^{\beta}$ are defined in (48) and $\gamma_{\delta}(\xi)=\left\{\xi+\delta e^{\imath \theta}, \theta \in\right] 0, \pi[\}$ (semi circle of centre $\xi$ and radius $\delta$ ) and $\Gamma_{\omega, \delta, \beta}$ is oriented according to Figure 8.

If we chooze $\beta>0$, small enough, we know that $\Gamma_{\omega, \delta, \beta} \subset D_{\omega}$ and does not contain the poles $\{\xi \in \Xi(\omega)\}$.

Therefore, we can use the Cauchy formula

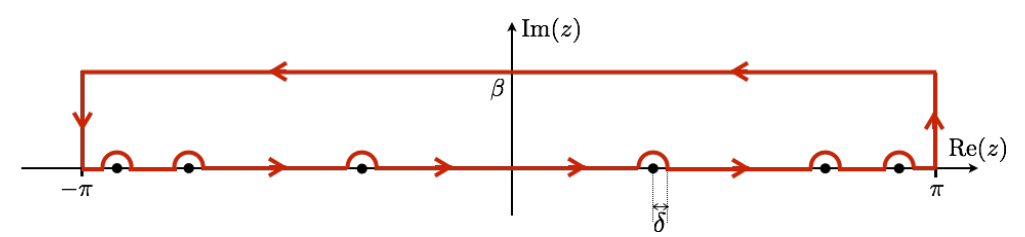

Fig. 8 Oriented contour $\Gamma_{\omega, \delta, \beta}$

$$
\int_{\Gamma_{\omega, \delta, \beta}} \hat{F}_{\omega}(\cdot ; z) e^{\imath p z} d z=0
$$


to deduce that

$$
\begin{aligned}
u_{\text {prop }}^{*}(\cdot+(p, 0)) & =w_{\text {prop }}^{+}(\cdot+(p, 0)) \\
& -\frac{1}{\sqrt{2 \pi}} \sum_{ \pm} \int_{S_{ \pm}^{\beta}}\left(A_{p}(k)-\omega^{2}\right)^{-1} \mathbb{P}_{\omega}(k) \hat{f}(\cdot ; k) e^{\imath p k} d k \\
& +\frac{1}{\sqrt{2 \pi}} \lim _{\delta \rightarrow 0} \sum_{n \in I(\omega)} \sum_{\xi \in \Xi_{n}(\omega)} \int_{\gamma_{\delta}(\xi)} \frac{\mathbb{P}_{n}(f)(\cdot ; z)}{\mu_{n}(z)-\omega^{2}} e^{\imath p z} d z
\end{aligned}
$$

where, proceeding as in the proof of Lemma 4

$$
w_{\text {prop }}^{+}(\cdot+(p, 0))=e^{-p \beta} \frac{1}{\sqrt{2 \pi}} \int_{-\pi}^{\pi} \mathbb{R}_{\omega}^{\text {prop }}(k+\imath \beta) \hat{f}(\cdot ; k+\imath \beta) e^{\imath p k} d k
$$

where we have set

$$
\mathbb{R}_{\omega}^{\text {prop }}(z):=\left(A_{p}(z)-\omega^{2}\right)^{-1} \mathbb{P}_{\omega}(z), \quad \forall z \in D_{\omega} \backslash \Xi(\omega)
$$

Setting $C_{\beta}^{\text {prop }}:=\sup _{z \in S^{\beta}}\left\|\mathbb{R}_{\omega}^{\text {prop }}(z)\right\|_{\mathcal{L}\left(L^{2}(\mathcal{C}), H^{1}(\triangle, \mathcal{C})\right)}$, we thus have

$$
\left\|w_{\text {prop }}^{+}\right\|_{H^{1}\left(\triangle, \mathcal{C}_{p}\right)} \leq C_{\beta}^{\text {prop }} e^{-p \beta} \sup _{z \in S^{\beta}}\|\hat{f}(\cdot ; z)\|_{L^{2}(\mathcal{C})} .
$$

For the last term (50), we use a well-known generalization of the Residue Theorem: for any function $g$ meromorphic in the neighborhood of $\xi$, which is supposed to be a simple pole, we have

$$
\lim _{\delta \rightarrow 0} \int_{\gamma_{\delta}(\xi)} g(z) d z=\imath \pi \lim _{z \rightarrow \xi}(z-\xi) g(z)
$$

Since $\omega \notin \sigma_{0}$, for all $\xi \in \Xi_{n}(\omega), \mu_{n}^{\prime}(\xi) \neq 0$, so that $\xi$ is a simple pole of

$$
\frac{\mathbb{P}_{n}(f)\left(x_{1}, x_{s} ; z\right)}{\mu_{n}(z)-\omega^{2}} e^{\imath p z}
$$

and we deduce that

$$
\forall \xi \in \Xi_{n}(\omega), \quad \lim _{\delta \rightarrow 0} \int_{\gamma_{\delta}(\xi)} \frac{\mathbb{P}_{n}(f)\left(x_{1}, x_{s} ; z\right)}{\mu_{n}(z)-\omega^{2}} e^{\imath p z} d z=\imath \pi \frac{\mathbb{P}_{n}(f)\left(x_{1}, x_{s} ; \xi\right)}{\mu_{n}^{\prime}(\xi)} e^{\imath p \xi}
$$

This completes the proof for the first formula. The proof for the second formula is obtained by changing the contour $\Gamma_{\omega, \delta, \beta}$ by its symmetric with respect to the real axis, which in particular changes $\imath \pi$ into $-\imath \pi$ in the formula (51).

In addition to Lemmas 4 and 5, the third argument of the proof of our main result will be the following lemma that essentially expresses a consequence of the $2 \pi$ periodicity of the operator $A_{p}(k)$. Without any loss of generality (see Remark 2), we assume that

$$
\pi \text { and }-\pi \text { do not belong to } \Xi(\omega) .
$$


Lemma 6 As soon as $\beta$ is small enough, one has the identities

$$
\begin{aligned}
& \int_{\pi}^{\pi+\imath \beta}\left(A_{p}(k)-\omega^{2}\right)^{-1} \mathbb{P}_{\omega}(k) \hat{f}(\cdot ; k) e^{\imath p k} d k \\
& \quad+\int_{\pi}^{\pi+\imath \beta}\left(A_{p}(k) \mathbb{Q}_{\omega}(k)-\omega^{2}\right)^{-1} \mathbb{Q}_{\omega}(k) \hat{f}(\cdot ; k) e^{\imath p k} d k= \\
& \int_{-\pi}^{-\pi+\imath \beta}\left(A_{p}(k)-\omega^{2}\right)^{-1} \mathbb{P}_{\omega}(k) \hat{f}(\cdot ; k) e^{\imath p k} d k \\
& \quad+\int_{-\pi}^{-\pi+\imath \beta}\left(A_{p}(k) \mathbb{Q}_{\omega}(k)-\omega^{2}\right)^{-1} \mathbb{Q}_{\omega}(k) \hat{f}(\cdot ; k) e^{\imath p k} d k \\
& \int_{\pi-\imath \beta}^{\pi}\left(A_{p}(k)-\omega^{2}\right)^{-1} \mathbb{P}_{\omega}(k) \hat{f}(\cdot ; k) e^{\imath p k} d k \\
& \quad+\int_{\pi-\imath \beta}^{\pi}\left(A_{p}(k) \mathbb{Q}_{\omega}(k)-\omega^{2}\right)^{-1} \mathbb{Q}_{\omega}(k) \hat{f}(\cdot ; k) e^{\imath p k} d k= \\
& \int_{\pi-\imath \beta}^{\pi}\left(A_{p}(k)-\omega^{2}\right)^{-1} \mathbb{P}_{\omega}(k) \hat{f}(\cdot ; k) e^{\imath p k} d k \\
& +\int_{\pi-\imath \beta}^{\pi}\left(A_{p}(k) \mathbb{Q}_{\omega}(k)-\omega^{2}\right)^{-1} \mathbb{Q} \omega(k) \hat{f}(\cdot ; k) e^{\imath p k} d k
\end{aligned}
$$

Proof Since $\pi$ and $-\pi$ do not belong to $\Xi(\omega), A_{p}( \pm \pi)-\omega^{2}$ is invertible. Thus, by perturbation theory again, there exists $\rho>0$ such that:

- the two balls $B_{\rho}^{ \pm}=\{k,|k \mp \pi|<\rho\}$ are included in $D_{\omega}$ and $B_{\rho}^{ \pm} \cap \Xi(\omega)=\emptyset$;

$-A_{p}(k)-\omega^{2}$ is invertible in $|k- \pm \pi|<\rho$ and the resolvent

$$
k \mapsto\left(A_{p}(k)-\omega^{2}\right)^{-1}
$$

is bounded analytic in $B_{\rho}^{ \pm}$.

Moreover, from the $2 \pi$ periodicity of $A_{p}(k)$, we deduce the $2 \pi$ periodicity of its resolvent. In particular

$$
\text { For }|\lambda| \leq \rho, \quad\left(A_{p}(\pi+i \lambda)-\omega^{2}\right)^{-1}=\left(A_{p}(-\pi+i \lambda)-\omega^{2}\right)^{-1}
$$

Next, we observe that, for real values of $k$ such that $k \in[-\pi, \pi] \backslash \Xi(\omega)$

$$
\left(A_{p}(k)-\omega^{2}\right)^{-1} \mathbb{P}_{\omega}(k)+\left(A_{p}(k) \mathbb{Q}_{\omega}(k)-\omega^{2}\right)^{-1} \mathbb{Q}_{\omega}(k)=\left(A_{p}(k)-\omega^{2}\right)^{-1}
$$


It suffices to observe that, applied to any $\psi_{m}(k)$, the operators at, respectively, the left hand side and right hand side of (56) give the same value, namely

$$
\left(\mu_{m}(k)-\omega^{2}\right)^{-1} \psi_{m}(k)
$$

and use the fact that the $\psi_{m}(k)$ 's form an orthonormal basis of $L^{2}(\mathcal{C})$. The two terms respectively at the right hand side and left hand side of (56) are bounded analytic functions of $k$ in each ball $B_{\rho}^{ \pm}$. As they coincide along the segments $[-\pi,-\pi+\rho]$ and $[\pi-\rho, \pi]$ according to $(56)$, by analyticity they coincide everywhere in the balls $B_{\rho}^{ \pm}$, and in particular along the vertical segments $[-\pi-i \beta,-\pi+i \beta]$ and $[\pi-i \beta, \pi+i \beta]$ as soon as $\beta<\rho$.

As a consequence, the left and right hand sides of (53) are nothing but

$$
\int_{\pi}^{\pi+\imath \beta}\left(A_{p}(k)-\omega^{2}\right)^{-1} \hat{f}(\cdot ; k) e^{\imath p k} d k \quad \text { and } \quad \int_{-\pi}^{-\pi+\imath \beta}\left(A_{p}(k)-\omega^{2}\right)^{-1} \hat{f}(\cdot ; k) e^{\imath p k} d k
$$

which coincide thanks to the periodicity property (55). This proves (53). The same holds for (54).

We can now state our main result.

Theorem 7 (Asymptotic behaviour of the solution at $\pm \infty$ )

$$
\begin{array}{r}
u(\cdot+(p, 0))=\imath \sqrt{2 \pi} \sum_{n \in I(\omega)} \sum_{\xi \in \Xi_{n}^{ \pm}(\omega)} \frac{\left(\hat{f}(\cdot ; \xi), \psi_{n}(\cdot ; \xi)\right)_{L^{2}(\mathcal{C})}}{\left|\mu_{n}^{\prime}(\xi)\right|} \psi_{n}(\cdot ; \xi) e^{\imath p \xi} \\
+w^{ \pm}(\cdot+(p, 0))
\end{array}
$$

where $w^{+}$(resp. $\left.w^{-}\right)$is exponentially decaying in $H_{l o c}^{1}(\triangle, \Omega)$ at $+\infty$ (resp. $-\infty)$.

Proof Collecting the results of Lemmas 4, 5 and 6, we deduce from (42), (43), (44) and (45) that, setting $w^{ \pm}:=w_{\text {evan }}^{ \pm}+w_{\text {prop }}^{ \pm}$

$$
\begin{aligned}
u(\cdot+(p, 0)) & =\imath \sqrt{\frac{\pi}{2}}\left(\sum_{\xi \in \Xi_{n}(\omega)} \frac{\mathbb{P}_{n}(f)(\cdot ; \xi)}{\left|\mu_{n}^{\prime}(\xi)\right|} e^{\imath p \xi} \pm \sum_{\xi \in \Xi_{n}(\omega)} \frac{\mathbb{P}_{n}(f)(\cdot ; \xi)}{\mu_{n}^{\prime}(\xi)} e^{\imath p \xi}\right) \\
& +w^{ \pm}(\cdot+(p, 0))
\end{aligned}
$$

Then it suffices to remark that, by definition of $\Xi_{n}^{ \pm}(\omega)$, with the + sign, the sum over $\Xi_{n}^{+}(\omega)$ is doubled and the sums over $\Xi_{n}^{-}(\omega)$ cancel while the contrary occurs with the - sign.

Physically, this theorem means that the solution $u$ behaves when $x_{1}$ tends to $+\infty$ (resp. to $-\infty$ ) as a linear combination of the Floquet modes $\psi_{n}(x, \xi)$, $n \in I(\omega), \xi \in \Xi_{n}^{+}(\omega)$ (resp. $\psi_{n}(x, \xi), n \in I(\omega), \xi \in \Xi_{n}^{-}(\omega)$ ) which propagate to the right (resp. to the left) as $\mu_{n}^{\prime}(\xi)>0$ (resp. $\mu_{n}(\xi)=\omega^{2}$ and $\left.\mu_{n}^{\prime}(\xi)<0\right)$. 
Similarly, the solution $u$ behaves when $x_{1}$ tends to $-\infty$ as a linear combination of the Floquet modes $\psi_{n}(x, \xi), n \in I(\omega), \xi \in \Xi_{n}^{-}(\omega)$ which propagate to the left as $\mu_{n}^{\prime}(\xi)<0$. In particular, when $\omega^{2}$ does not belong to $A_{p}, \Xi(\omega)$ is empty and the theorem expresses the fact that the solution is exponentially decreasing at $\pm \infty$.

Remark 9 We can deduce the asymptotic behaviour at $\pm \infty$ of the Green function $G$ defined in Remark 8. Suppose that $\omega \notin \sigma_{0}$, for all $\left(x_{1}, x_{s}\right) \in \mathcal{C},\left(y_{1}, y_{s}\right) \in$ $\mathcal{C}, p, q \in \mathbb{Z}$

$$
\begin{gathered}
G\left(x_{1}+p, x_{s} ; y_{1}+q, y_{s}\right)=\imath \sum_{n \in I(\omega)} \sum_{\xi \in \Xi_{n}^{ \pm}(\omega)} \frac{\psi_{n}\left(x_{1}, x_{s} ; \xi\right) \overline{\psi_{n}\left(y_{1}, y_{s} ; \xi\right)}}{\left|\mu_{n}^{\prime}(\xi)\right|} e^{\imath(p-q) \xi} \\
+w^{ \pm}\left(x_{1}+p, x_{s}\right)
\end{gathered}
$$

where $w^{+}$(resp. $w^{-}$) is exponentially decaying at $+\infty$ (resp. $\left.-\infty\right)$.

For the sequel, and in order to simplify the expression of our result, let us introduce a renumbering of the set $\Xi^{+}(\omega)$ defined in (23)

$$
\Xi^{+}(\omega)=\bigcup_{n \in I(\omega)} \Xi_{n}^{+}(\omega)=\left\{\xi_{1}^{+} \leq \ldots \leq \xi_{\ell}^{+} \leq \ldots \leq \xi_{N(\omega)}^{+}\right\}
$$

where $N(\omega)=\# \Xi^{+}(\omega)$ is the number of Floquet modes propagating to the right. Thanks to Proposition 4, we have

$$
\Xi^{-}(\omega)=\bigcup_{n \in I(\omega)} \Xi_{n}^{-}(\omega)=\left\{-\xi_{1}^{+} \geq \ldots \geq-\xi_{\ell}^{+} \geq \ldots \geq-\xi_{N(\omega)}^{+}\right\}
$$

We renumber accordingly the set $\left.\left\{\psi_{n}(\cdot ; \xi), \xi \in \Xi_{n}^{+}(\omega), n \in I(\omega)\right)\right\}$ and renormalize them for convenience of notation. More precisely, we introduce $\phi_{1}, \ldots, \phi_{N(\omega)}$ respectively $\phi_{-1}, \ldots, \phi_{-N(\omega)}$ defined as

$$
\forall n \in I(\omega), \forall \xi \in \Xi_{n}^{ \pm}(\omega), \quad \xi= \pm \xi_{m}^{+} \Rightarrow \phi_{ \pm m}=\frac{\psi_{n}(\cdot ; \xi)}{\sqrt{\left|\mu_{n}^{\prime}(\xi)\right|}}
$$

Then the result of Theorem 7 becomes

$$
u(\cdot+(p, 0))=\imath \sqrt{2 \pi} \sum_{m=1}^{N(\omega)}\left(\hat{f}\left(\cdot ; \pm \xi_{m}^{+}\right), \phi_{ \pm m}\right)_{\mathcal{C}} \phi_{ \pm m} e^{ \pm \imath p \xi_{m}^{+}}+w^{ \pm}(\cdot+(p, 0))
$$

where $w^{+}$(resp. $w^{-}$) is exponentially decaying in $H_{\mathrm{loc}}^{1}(\triangle, \Omega)$ at $+\infty$ (resp. $-\infty)$.

Remark 10 In the Appendix B, we compute the far field of the fundamental solution in the special case of the straight homogeneous waveguide, that is $n_{p}=1$ in $\Omega=\mathbb{S} \times \mathbb{R}$. In particular, we retrieve the expression obtained by using directly the well-known fundamental solution for the homogeneous waveguide. 


\section{Radiation condition and uniqueness of the solution}

Thanks to the main result of the last section (see Theorem 7), we can define a radiation condition and establish the well-posedness of the Helmholtz equation set in a periodic waveguide.

Definition 5 (The outgoing radiation condition) We say that $u$ satisfies the outgoing radiation condition if and only if there exist $N(\omega)$ complex numbers $\left(u_{n}^{ \pm}\right)_{n \in \llbracket 1, N(\omega) \rrbracket}$ such that

$$
u(\cdot+(p, 0))=\sum_{m=1}^{N(\omega)} u_{m}^{ \pm} \phi_{ \pm m} e^{ \pm \imath p \xi_{m}^{+}}+w^{ \pm}(\cdot+(p, 0))
$$

where $w^{+}$(resp. $w^{-}$) is exponentially decaying in $H_{\text {loc }}^{1}(\triangle, \Omega)$ at $+\infty$ (resp. $-\infty)$.

Theorem 8 (Well-posedness of the problem) Suppose $\omega^{2} \notin \sigma_{0}$. There exists a unique solution of problem (1) which satisfies the outgoing radiation condition.

Proof Thanks to Theorems 6 and 7, we have shown that the function given by (37-38) is a solution to problem (1) satisfying the outgoing radiation condition. It remains to show the uniqueness of such a solution. Let $u$ be a solution of

$$
\begin{cases}-\triangle u-n_{p}^{2} \omega^{2} u=0 & \text { in } \Omega \\ \nabla u \cdot \mathbf{n}=0 & \text { on } \partial \Omega\end{cases}
$$

which satisfies the outgoing radiation condition, that is there exists $\left(u_{n}^{ \pm}\right)_{n \in \llbracket 1, N(\omega) \rrbracket}$

$$
u(\cdot+(p, 0))=\sum_{m=1}^{N(\omega)} u_{m}^{ \pm} \phi_{ \pm m} e^{ \pm \imath p \xi_{m}^{+}}+w^{ \pm}(\cdot+(p, 0))
$$

where $w^{+}$(respectively $w^{-}$) is exponentially decaying in $H_{\mathrm{loc}}^{1}(\triangle, \Omega)$ at $+\infty$ (respectively $-\infty$ ). Let us show that $u=0$.

We first notice that $u \in \mathcal{V}(\omega)$ defined in (26). By using the property (29) for $x=-N$ and $x=N$ for some $N \in \mathbb{N}^{*}$

$$
q(-N, u, u)=q(N, u, u)
$$


We now use the formula $\left(R C^{ \pm}\right)$on $\Gamma_{ \pm N}=\left\{x_{s},\left( \pm N, x_{s}\right) \in \Omega\right\}$

$$
\begin{aligned}
q( \pm N, u, u) & =\sum_{n, m=1}^{N(\omega)} u_{n}^{ \pm} \bar{u}_{m}^{ \pm} q\left(\phi_{ \pm n}, \phi_{ \pm m}\right) \\
& +\sum_{n=1}^{N(\omega)}\left[u_{n}^{ \pm} q\left( \pm N, \phi_{ \pm n}, w^{ \pm}\right)+\bar{u}_{n}^{ \pm} q\left( \pm N, w^{ \pm}, \phi_{ \pm n}\right)\right]+q\left( \pm N, w^{ \pm}, w^{ \pm}\right) \\
& = \pm \imath \sum_{n}^{N(\omega)}\left|u_{n}^{ \pm}\right|^{2} \\
& +\sum_{n=1}^{N(\omega)}\left[u_{n}^{ \pm} q\left( \pm N, \phi_{ \pm n}, w^{ \pm}\right)+\bar{u}_{n}^{ \pm} q\left( \pm N, w^{ \pm}, \phi_{ \pm n}\right)\right]+q\left( \pm N, w^{ \pm}, w^{ \pm}\right)
\end{aligned}
$$

thanks to Proposition 3 and Definition (59). Using the exponential decay property of $w^{ \pm}$, we know, in particular, that

$$
\left\|w^{ \pm}\right\|_{L^{2}\left(\Gamma_{ \pm N}\right)}+\left\|\frac{\partial w^{ \pm}}{\partial x_{1}}\right\|_{L^{2}\left(\Gamma_{ \pm N}\right)} \leq C e^{-\alpha N}
$$

for some $\alpha>0$ while by quasi-periodicity it is clear that

$$
\forall n \in \llbracket 1, N(\omega) \rrbracket, \quad\left\|\phi_{ \pm n}\right\|_{L^{2}\left(\Gamma_{ \pm N}\right)}+\left\|\frac{\partial \phi_{ \pm n}}{\partial x_{1}}\right\|_{L^{2}\left(\Gamma_{ \pm N}\right)} \leq C
$$

We deduce that

$$
\lim _{N \rightarrow+\infty} q( \pm N, u, u)= \pm \imath \sum_{n}^{N(\omega)}\left|u_{n}^{ \pm}\right|^{2}
$$

Taking the limit when $N$ tends to $+\infty$ in the equality (62), we conclude that

$$
\forall n \in \llbracket 1, N(\omega) \rrbracket, \quad u_{n}^{ \pm}=0 .
$$

The function $u$ is then a solution of (61) which is exponentially decaying and thus belongs to the domain of the operator $A$. If $u$ was not 0 , this would mean that $u$ is an eigenvector of $A_{p}$ with eigenvalue $\omega^{2}$ but this is impossible since $\omega^{2} \notin \sigma_{0}$.

Remark 11 The reader will note that the existence and uniqueness result of Theorem 8 remains valid under the weaker notion of a radiation condition where in the Definition 5 the exponential decay property of $w^{ \pm}$is simply replaced by

$$
\lim _{N \rightarrow+\infty}\left[\left\|w^{ \pm}\right\|_{L^{2}\left(\Gamma_{ \pm N}\right)}+\left\|\frac{\partial w^{ \pm}}{\partial x_{1}}\right\|_{L^{2}\left(\Gamma_{ \pm N}\right)}\right]=0
$$

The exponential decay of $w^{ \pm}$appears then as an additional property of the outgoing solution. 


\section{A Proof of Theorem 6}

Let us write $u_{\varepsilon}=u_{\text {evan }}^{\varepsilon}+u_{\text {prop }}^{\varepsilon}$ where

$$
u_{\mathrm{evan}}^{\varepsilon}=\sum_{n \notin I(\omega)} u_{\varepsilon}^{n} \text { and } u_{\text {prop }}^{\varepsilon}=\sum_{n \in I(\omega)} u_{\varepsilon}^{n} .
$$

We show separately that $u_{\text {evan }}^{\varepsilon}$ tends to $u_{\text {evan }}$ in $H^{1}(\Omega)$ (and implicitly that $u_{\text {evan }}$ is in $\left.H^{1}(\Omega)\right)$ and that $u_{\text {prop }}^{\varepsilon}$ tends to $u_{\text {prop }}$ in $H_{\text {loc }}^{1}(\Omega)$ when $\varepsilon$ goes to 0 where $u_{\text {evan }}$ and $u_{\text {prop }}$ are defined in (37-38).

Step 1: $\left\|u_{\text {evan }}^{\varepsilon}-u_{\text {evan }}\right\|_{H^{1}(\Omega)} \leq C(\omega) \varepsilon\|f\|_{L^{2}(\Omega)}$

We set

$$
\forall k \in[-\pi, \pi], \quad d_{\varepsilon}^{n}(k)=\left(\mu_{n}(k)-\left(\omega^{2}+\imath \varepsilon\right)\right)^{-1}-\left(\mu_{n}(k)-\omega^{2}\right)^{-1}
$$

that satisfies

$$
\forall k \in[-\pi, \pi], \quad\left|d_{\varepsilon}^{n}(k)\right| \leq \frac{\varepsilon}{\left|\mu_{n}(k)-\omega^{2}\right|^{2}}
$$

and by definition of the set $I(\omega)$,

$$
\inf _{n \notin I(\omega)} \inf _{k \in[-\pi, \pi]}\left|\mu_{n}(k)-\omega^{2}\right|>0
$$

from which we deduce that

$$
\forall k \in[-\pi, \pi], \quad\left|d_{\varepsilon}^{n}(k)\right| \leq C_{0}(\omega) \varepsilon .
$$

Using Proposition 1, we have

$$
\left\|u_{\text {evan }}^{\varepsilon}-u_{\text {evan }}\right\|_{n_{p}^{2}, \Omega}^{2}=\int_{-\pi}^{\pi}\left\|\hat{u}_{\text {evan }}^{\varepsilon}(\cdot ; k)-\hat{u}_{\text {evan }}(\cdot ; k)\right\|_{n_{p}^{2}, \mathcal{C}}^{2} d k
$$

where each $k \in[-\pi, \pi], \hat{u}_{\text {evan }}^{\varepsilon}(\cdot ; k)$ and $\hat{u}_{\text {evan }}^{\varepsilon}(\cdot ; k)$ are given by

$$
\hat{u}_{\text {evan }}^{\varepsilon}(\cdot ; k)=\sum_{n \notin I(\omega)} \frac{\mathbb{P}_{n}(f)(\cdot, k)}{\mu_{n}(k)-\left(\omega^{2}+i \varepsilon\right)}, \quad \hat{u}_{\text {evan }}(\cdot ; k)=\sum_{n \notin I(\omega)} \frac{\mathbb{P}_{n}(f)(\cdot, k)}{\mu_{n}(k)-\omega^{2}}
$$

From the orthogonality in $L^{2}\left(\mathcal{C}, n_{p}^{2} d x\right)$ of the functions $\mathbb{P}_{n}(f)(\cdot, k)$, we deduce that

$$
\left\|\hat{u}_{\text {evan }}^{\varepsilon}(\cdot ; k)-\hat{u}_{\text {evan }}(\cdot ; k)\right\|_{n_{p}^{2}, \mathcal{C}}^{2}=\sum_{n \notin I(\omega)}\left|d_{\varepsilon}^{n}(k)\right|^{2}\left|\left(\hat{f}(\cdot ; k), \psi_{n}(\cdot ; k)\right)_{n_{p}^{2}, \mathcal{C}}\right|^{2} \leq C_{0}(\omega)^{2} \varepsilon^{2}\|\hat{f}(\cdot ; k)\|_{n_{p}^{2}, \mathcal{C}}^{2}
$$

That is to say

$$
\left\|u_{\text {evan }}^{\varepsilon}-u_{\text {evan }}\right\|_{n_{p}^{2}, \Omega} \leq C_{0}(\omega) \varepsilon\|f\|_{n_{p}^{2}, \Omega} .
$$

In the same way,

$$
\left\|\nabla u_{\text {evan }}^{\varepsilon}-\nabla u_{\text {evan }}\right\|_{\Omega}^{2}=\int_{-\pi}^{\pi}\left\|\nabla \hat{u}_{\text {evan }}^{\varepsilon}(\cdot ; k)-\nabla \hat{u}_{\text {evan }}(\cdot ; k)\right\|_{\mathcal{C}}^{2} d k
$$

and

$$
\left\|\nabla \hat{u}_{\mathrm{evan}}^{\varepsilon}(\cdot ; k)-\nabla \hat{u}_{\mathrm{evan}}(\cdot ; k)\right\|_{\mathcal{C}}^{2}=\sum_{n \notin I(\omega)}\left|\mu_{n}(k)\right|\left|d_{\varepsilon}^{n}(k)\right|^{2}\left|\left(\hat{f}(\cdot ; k), \psi_{n}(\cdot ; k)\right)_{n_{p}^{2}, \mathcal{C}}\right|^{2}
$$

One easily concludes, after having noticed that

$$
\forall k \in[-\pi, \pi], \quad\left|\mu_{n}(k)\right|\left|d_{\varepsilon}^{n}(k)\right|^{2} \leq C_{1}(\omega)^{2} \varepsilon^{2} .
$$


Step 2: For all $n \in I(\omega)$, for all $p \in \mathbb{Z}$ and for all $\eta \in(0,1)$

$$
\left\|u_{n}^{\varepsilon}-u_{n}\right\|_{H^{1}\left(C_{p}\right)} \leq C_{\eta}(\omega) \varepsilon^{1-\eta}\|f\|_{L^{2}(\Omega)}
$$

To prove the above equality, we shall use the following technical lemma, which is obtained by combining the Sokhotski-Plemelj theorem $[34,39]$ and the Sobolev continuous embedding $\left.\left.H^{r}(]-a, b[) \hookrightarrow C^{0, r-1 / 2}(]-a, b\right]\right)$ for $\left.r \in\right] 1 / 2,3 / 2[[1]$.

Lemma 7 Let $a, b>0, r \in] 1 / 2,3 / 2\left[\right.$ and $X$ a Banach space. For any $\psi \in H^{r}(]-a, b[, X)$, we define for any $\varepsilon>0$

$$
S_{\varepsilon} \psi=\int_{-a}^{b} \frac{\psi(t)}{t-\imath \varepsilon} d t \in X \quad \text { and } \quad S \psi=p . v . \int_{-a}^{b} \frac{\psi(t)}{t} d t+\psi(0) \in X
$$

then for $\varepsilon$ small enough, there exists a constant $C_{r}$ such that

$$
\forall \psi \in H^{r}(]-a, b[, X), \quad\left\|S_{\varepsilon} \psi-S \psi\right\|_{X} \leq C_{r} \varepsilon^{r-1 / 2}\|\psi\|_{H^{r}(]-a, b[, X)}
$$

Since $\omega^{2} \notin \sigma_{0}, \mu_{n}^{\prime}(\xi) \neq 0$ for all $\xi \in \Xi_{n}(\omega)$. Therefore one can fix a $\delta>0$ small enough such that $\mu_{n}(k)$ is monotonous in each of the (disjoint) intervals \{]$\xi-\delta, \xi+\delta\left[, \xi \in \Xi_{n}(\omega)\right\}$. Let us set

$$
\left.\mathcal{I}_{\omega, n}^{\delta}=\right]-\pi, \pi\left[\backslash \bigcup_{\xi \in \Xi_{n}(\omega)}\right] \xi-\delta, \xi+\delta[
$$

With this notation, we have $u_{n}^{\varepsilon}=u_{n}^{\varepsilon, \delta}+\sum_{\xi \in \Xi_{n}(\omega)} v_{n}^{\varepsilon, \delta}(\xi ; \cdot)$ and $u_{n}=u_{n}^{\delta}+\sum_{\xi \in \Xi_{n}(\omega)} v_{n}^{\delta}(\xi ; \cdot)$ where

$$
\begin{aligned}
\sqrt{2 \pi} u_{n}^{\varepsilon, \delta}\left(x_{1}+p, x_{s}\right) & =\int_{\mathcal{I}_{\omega, n}^{\delta}} \frac{\mathbb{P}_{n}(f)\left(x_{1}, x_{s} ; k\right)}{\mu_{n}(k)-\left(\omega^{2}+\imath \varepsilon\right)} e^{\imath p k} d k \\
\sqrt{2 \pi} u_{n}^{\delta}\left(x_{1}+p, x_{s}\right) & =\int_{\mathcal{I}_{\omega, n}^{\delta}} \frac{\mathbb{P}_{n}(f)\left(x_{1}, x_{s} ; k\right)}{\mu_{n}(k)-\omega^{2}} e^{\imath p k} d k \\
\sqrt{2 \pi} v_{n}^{\varepsilon, \delta}\left(\xi ; x_{1}+p, x_{s}\right) & =\int_{\xi-\delta}^{\xi+\delta} \frac{\mathbb{P}_{n}(f)\left(x_{1}, x_{s} ; k\right)}{\mu_{n}(k)-\left(\omega^{2}+\imath \varepsilon \omega\right)} e^{\imath p k} d k \\
\sqrt{2 \pi} v_{n}^{\delta}\left(\xi ; x_{1}+p, x_{s}\right) & =\operatorname{p.v} \int_{\xi-\delta}^{\xi+\delta} \frac{\mathbb{P}_{n}(f)\left(x_{1}, x_{s} ; k\right)}{\mu_{n}(k)-\omega^{2}} e^{\imath p k} d k+\imath \pi \frac{\mathbb{P}_{n}(f)\left(x_{1}, x_{s} ; \xi\right)}{\left|\mu_{n}^{\prime}(\xi)\right|}
\end{aligned}
$$

Proceeding along the same lines as in Step 1, it can be proven immediately that

$$
\left\|u_{n}^{\varepsilon, \delta}-u_{n}^{\delta}\right\|_{H^{1}(\Omega)} \leq C(\omega, \delta) \varepsilon\|f\|_{L^{2}(\Omega)} .
$$

It remains to estimate $v_{n}^{\varepsilon, \delta}(\xi ; \cdot)-v_{n}^{\delta}(\xi ; \cdot)$ for each $\xi \in \Xi_{n}(\omega)$. Thanks to our choice of $\delta$ and since $\mu_{n}(\xi)=\omega^{2}$, the function $k \mapsto \mu_{n}(k)$ is invertible from $] \xi-\delta, \xi+\delta$ [ onto ]$-a_{n}(\xi)+\omega^{2}, b_{n}(\xi)+\omega^{2}$ [ with $a_{n}(\xi), b_{n}(\xi)>0$. We perform the change of variable $k=$ $\nu_{n}\left(t+\omega^{2}\right)$ where $\nu_{n}=\mu_{n}^{-1}$ and we obtain

$$
\sqrt{2 \pi} v_{n}^{\varepsilon, \delta}(\xi ; \cdot)=\int_{-a_{n}(\xi)}^{b_{n}(\xi)} \frac{\phi_{n}(\cdot ; t)}{t-\imath \varepsilon} d t
$$

where

$$
\forall\left(x_{1}, x_{s}\right) \in \mathcal{C}, \forall p \in \mathbb{Z}, \quad \phi_{n}\left(x_{1}+p, x_{s} ; t\right)=\mathbb{P}_{n}(f)\left(x_{1}, x_{s} ; \nu_{n}\left(t+\omega^{2}\right)\right) \frac{e^{\imath p \nu_{n}\left(t+\omega^{2}\right)}}{\left|\mu_{n}^{\prime}\left(\nu_{n}\left(t+\omega^{2}\right)\right)\right|}
$$

In the same way, we can write

$$
\sqrt{2 \pi} v_{n}^{\varepsilon, \delta}(\xi ; \cdot)=\text { p.v. } \int_{-a_{n}(\xi)}^{b_{n}(\xi)} \frac{\phi_{n}(\cdot ; t)}{t} d t+\imath \pi \psi_{n}(\cdot ; 0) .
$$


To conclude, we wish to apply Lemma 7 with $X=H^{1}(\mathcal{C})$. Then it suffices to remark that

$$
\phi_{n} \in H^{r}(]-a_{n}(\xi), b_{n}(\xi)\left[, H^{1}(\mathcal{C})\right) \quad \text { for any } r>0 .
$$

Indeed, since $f$ is of compact support, we know that

$$
\|f\|_{L_{r}^{2}(\Omega)}^{2}=\int_{\Omega}\left|f\left(x_{1}, x_{s}\right)\right|^{2}\left(1+x_{1}^{2}\right)^{r} d x_{1} d x_{s}<+\infty \quad \text { for any } r>0,
$$

so using the property of the Floquet Bloch Transform $[22,11]$, we show that

$$
\hat{f} \in H^{r}(]-\pi, \pi\left[, L^{2}(\mathcal{C})\right) \text { for any } r>0 .
$$

More precisely, exploiting the $C^{\infty}$ regularity of $\nu_{n}\left(t+\omega^{2}\right), \mu_{n}^{\prime}(t)$ and $\phi_{n}\left(\cdot ; \nu_{n}\left(t+\omega^{2}\right)\right)$, it is not difficult (the details are left to the reader) to show that

$$
\exists C(\omega), \quad\left\|\psi_{n}\right\|_{H^{r}(]-a_{n}(\xi), b_{n}(\xi)\left[, H^{1}(\mathcal{C})\right)} \leq C(\omega)\|f\|_{L_{r}^{2}(\Omega)}
$$

where

$$
\|f\|_{L_{r}^{2}(\Omega)} \leq\|f\|_{L^{2}(\Omega)}
$$

because $f$ is of compact support.

Remark 12 Let us remark (this is classical in scattering theory) that the result of the theorem does not require that $f$ has compact support but simply that $f$ belongs to a $L_{r}^{2}$ for $r>1 / 2$. If $r \geq 3 / 2$, then the result (63) holds and if $1 / 2<r<3 / 2$, one has to replace $1-\eta$ in the estimation (63) by $r-1 / 2$.

\section{B The homogeneous waveguide as a particular instance of periodic waveguides}

The homogeneous waveguide is obviously a particular case of periodic waveguides for which $n_{p}=1$ in $\Omega=\mathbb{S} \times \mathbb{R}$.

Firstly, let us see what the propagating Floquet modes in that case are. For this purpose we introduce the so-called guided modes, which appear when we investigate the solutions of

$$
\left\{\begin{array}{ccc}
-\triangle u-\omega^{2} u=0 & \text { in } \quad \Omega \\
\nabla u \cdot \mathbf{n}=0 & \text { on } & \partial \Omega
\end{array}\right.
$$

Indeed, these solutions are the linear combinations of the guided modes given by

$$
u_{m}^{ \pm}\left(x_{1}, x_{2}\right)=\theta_{m}\left(x_{s}\right) e^{ \pm i \beta_{m} x_{1}}, \quad m \geq 1,
$$

with

$$
\beta_{m}=\sqrt{\omega^{2}-\alpha_{m}}, \quad \operatorname{Re}\left(\beta_{\mathrm{m}}\right), \operatorname{Im}\left(\beta_{m}\right) \geq 0,
$$

where $0=\alpha_{1} \leq \ldots \leq \alpha_{m} \rightarrow+\infty$ are the eigenvalues of the tranverse laplacian $-\triangle_{\mathbb{S}}$ with Neumann boundary conditions on $\partial \mathbb{S}$ and $\theta_{1}, \ldots, \theta_{m}, \ldots$ are associated eigenvectors (forming an orthonormal basis of $L^{2}(\mathbb{S})$ ). For example, if $\mathbb{S}=(0,1)$

$$
\alpha_{m}=(m-1)^{2} \pi^{2}
$$

and

$$
\left\{\begin{array}{c}
\theta_{1}\left(x_{s}\right)=1 \\
\theta_{m}\left(x_{s}\right)=\sqrt{2} \cos \left((m-1) \pi x_{s}\right) \quad(m \geq 2) .
\end{array}\right.
$$

Among the guided modes, the propagating guided modes correspond to the purely real $\beta_{m}$. Because the sequence $\left(\alpha_{m}\right)_{m}$ tends to $+\infty$, there is only a finite number of propagative 
modes - for example for $\mathbb{S}=(0,1)$, there are only $2 P$ propagative modes with $P:=C(k / \pi)$, where $C$ is the ceiling function. The other guided modes are either increasing or decreasing exponentially with variable $x_{1}$. Let us verify that the two notions, propagating Floquet modes in the homogeneous case and propagating guided modes, do coincide. It is easy to show that the eigenvalues and eigenfunctions of operator $A_{p}(k)$ for $n_{p}=1$ are given, for all $m \geq 1$ and $l \in \mathbb{Z}$, by

$$
\mu_{m, l}(k)=\alpha_{m}+(k+2 \pi l)^{2}, \quad \psi_{m, l}(x ; k)=\theta_{m}\left(x_{s}\right) e^{\imath(k+2 \pi l) x_{1}} .
$$

Hence the $\lambda_{n}(k)$ coincide with the $\mu_{m, l}(k)$, up to a change of numbering. The propagating Floquet modes are then given, for $x \in \Omega$, by

$$
u_{m, l}(x ; \xi)=\theta_{m}\left(x_{s}\right) e^{i(\xi+2 \pi l) x_{1}},
$$

for all $\xi \in(-\pi, \pi], m \geq 1$ and $l \in \mathbb{Z}$ such that

$$
\alpha_{m}+(\xi+2 \pi l)^{2}=\omega^{2} .
$$

Let us consider a propagating Floquet mode $u_{m, l}(\cdot, \xi)$. Since $(71)$ is satisfied, then

$$
\xi+2 \pi l= \pm \sqrt{\omega^{2}-\alpha_{m}}= \pm \beta_{m}
$$

and therefore $u_{m, l}=u_{m}^{ \pm}$as given by (65), that is, $u_{m, l}$ is a propagating guided mode. Conversely, let us consider a propagating guided mode $u_{m}^{ \pm}$. There is a unique pair $(\xi, l)$ with $\xi \in(-\pi, \pi]$ and $l \in \mathbb{Z}$ such that $\pm \beta_{m}=\xi+2 \pi l$ (we denote $\xi= \pm \beta_{m} \bmod 2 \pi$ ). These $u_{m}^{ \pm}$ coincides with the propagating Floquet mode $u_{m, l}(\cdot, \xi)$ given by $(70)$. This completes the verification.

For some indices $n \in I(\omega)$ and $\xi \in \Xi_{n}(\omega)$, we associate the unique corresponding pair $(m, l)$ such that the propagating Floquet mode $u_{n}(\cdot ; \xi)=u_{m, l}(\cdot ; \xi)$ coincides with a guided mode $u_{m}^{+}$or $u_{m}^{-}$. We have then, for $n \in I(\omega), \Xi_{n}(\omega)=\left\{ \pm \beta_{m} \bmod 2 \pi\right\}$ and by using the expression of $\mu_{m, l}$ in (69) we obtain that for $\xi \in \Xi_{n}(\omega), \lambda_{n}^{\prime}(\xi)= \pm 2 \beta_{m}$.

Bfurthernore, the set $\sigma_{0}$ is simply given by

$$
\sigma_{0}=\left\{\sqrt{\alpha_{m}}, m \geq 1\right\}
$$

Remark 13 One particular property of periodic waveguides is that there may be band gaps in the spectrum; that is intervals of $\omega$ for which no propagating Floquet mode exists, in other words $I(\omega)=\emptyset$. More generally, the number of propagating Floquet modes does not necessarily increase with $\omega$. This is in contrast with the case of the homogeneous waveguide with Neumann boundary conditions, for which at least two propagating modes $u_{1}^{ \pm}$exist whatever $\omega$ is, and the number of propagating modes increases with $\omega$.

Secondly, let us see what becomes of expression (58) of the far field of the fundamental solution when the waveguide is homogeneous. By using the computations above we obtain that such a far field is given by

$$
G\left(x_{1}, x_{s} ; y_{1}, y_{s}\right) \underset{x_{1} \rightarrow \pm \infty}{\sim} i \sum_{m=1}^{P} \frac{u_{m}^{ \pm}\left(x_{1}, x_{s}\right) u_{m}^{\mp}\left(y_{1}, y_{s}\right)}{2 \beta_{m}}
$$

where the guided modes $u_{m}^{ \pm}$are given by (65) and the $\beta_{m}$ are given by (66), while $P$ is the number of propagating modes in each direction of propagation. Fortunately, we retrieve such an expression by considering directly the far field of the fundamental solution of the homogeneous waveguide, that is:

$$
G(x, y)=i \sum_{m=1}^{+\infty} \frac{e^{i \beta_{m}\left|x_{1}-y_{1}\right|}}{2 \beta_{m}} \theta_{m}\left(x_{s}\right) \theta_{m}\left(y_{s}\right)
$$




\section{Conclusion and Ongoing works}

In this paper, we have given a somewhat complete theory for time harmonic wave propagation in a periodic waveguide. Of course, this problem is quite academic but it opens the gate to the analysis of more realistic (from the point of view of applications) situations. The first and simplest extension is a case of a local perturbation of a periodic waveguide. Using compact perturbation theory, it should be straightforward to show, using the same radiation condition as in this paper, an existence and uniqueness result, except maybe for a countable set of frequencies located in the resolvent set of the unperturbed periodic operator which can accumulate only at the edges of the essential spectrum. Another extension, which is slightly similar to the previous, is the junction between two different periodic waveguides. A more intricate extension is the junction between an homogeneous and a periodic halfspace. This last situation seems really interesting in terms of applications to photonics (see for instance $[33,4,3])$.

Acknowledgements This work was partially supported by ANR project METAMATH ANR-11-MONU-0016.

\section{References}

1. Robert A Adams and John JF Fournier. Sobolev spaces, volume 140. Academic press, 2003.

2. Shmuel Agmon. Lectures on exponential decay of solutions of second-order elliptic equations: bounds on eigenfunctions of $N$-body Schrödinger operators, volume 29 of Mathematical Notes. Princeton University Press, Princeton, NJ, 1982.

3. Lombardet B., Dunbar L. A., Ferrini R., and Houdr R. A quantitative analysis of self-collimation effects in planar photonic crystals. J. of App. Phys., 99:096108-1-to-3, 2006 .

4. Momeni B., Huang J., Soltani M., Askari M., Mohammadi S., Rakhsandehroo M., and Adibi A. Compact wavelength demultiplexing using focusing negative index photonic crystal superprisms. Optics Express, 14(6):2413-2422, 2006.

5. F. Bentosela, P. Duclos, and P. Exner. Absolute continuity in periodic thin tubes and strongly coupled leaky wires. Letters in Mathematical Physics, 65:75-82, 2003.

6. Anne-Sophie Bonnet-Bendhia and Axel Tillequin. A limiting absorption principle for scattering problems with unbounded obstacles. Math. Methods Appl. Sci., 24(14):1089 $1111,2001$.

7. Laurent Bourgeois and Sonia Fliss. On the identification of defects in a periodic waveguide from far field data. Inverse Problems, 30:095004, 2014.

8. D Eidus. The limiting absorption and amplitude principles for the diffraction problem with two unbounded media. Communications in Mathematical Physics, 107(1):29-38, 1986.

9. Daniel M Èidus and Clyde D Hill. On the principle of limiting absorption. Technical report, DTIC Document, 1963.

10. S. Fliss and P. Joly. Exact boundary conditions for time-harmonic wave propagation in locally perturbed periodic media. Applied Numerical Mathematics, doi:10.1016/j.apnum.2008.12.013, 2008.

11. Sonia Fliss. Etude mathématique et numérique de la propagation des ondes dans un milieu pèriodique présentant un défaut. Ph.D. thesis. Ecole Doctorale de l'Ecole Polytechnique, Palaiseau, France, 2009.

12. Christian Gérard and Francis Nier. The Mourre theory for analytically fibered operators. J. Funct. Anal., 152(1):202-219, 1998.

13. Pierre Grisvard. Elliptic problems in nonsmooth domains, volume 69. SIAM, 2011.

14. $\mathrm{Vu}$ Hoang. The limiting absorption principle for a periodic semi-infinite waveguide. SIAM Journal of Applied Mathematics, 71(3):791-810, 2011.

15. Viorel Iftimie. Principe d'absorption limite pour l'opérateur de Laplace-Dirichlet sur un ouvert au bord périodique. Math. Rep. (Bucur.), 5(55)(3):239-249, 2003. 
16. Arne Jensen, Éric Mourre, and Peter Perry. Multiple commutator estimates and resolvent smoothness in quantum scattering theory. Ann. Inst. H. Poincaré Phys. Théor., 41(2):207-225, 1984.

17. John D. Joannopoulos, Robert D. Meade, and Joshua Winn N. Photonic Crystal Molding the Flow of Light. Princeton Univeristy Press, 1995.

18. S.G. Johnson and John D. Joannopoulos. Photonic Crystal - The road from theory to practice. Kluwer Acad. Publ., 2002.

19. P. Joly, J.-R. Li, and S. Fliss. Exact boundary conditions for periodic waveguides containing a local perturbation. Commun. Comput. Phys., 1(6):945-973, 2006.

20. I. Kachkovskii and N. Filonov. Absolute continuity of the spectrum of a periodic schrdinger operator in a multidimensional cylinder. Algebra i Analiz, 21:133-152, 2009.

21. T Kato. Perturbation theory for linear operators. Classics in Mathematics. SpringerVerlag, Berlin, 1995. Reprint of the 1980 edition.

22. P. Kuchment. Floquet theory for partial differential equations, volume 60 of Operator Theory: Advances and Applications. Birkhäuser Verlag, Basel, 1993.

23. P. Kuchment. The mathematics of photonic crystals (chapter 7). In Mathematical modeling in optical science, volume 22 of Frontiers in applied mathematics. SIAM, Philadelphia, 2001.

24. P. Kuchment. On some spectral problems of mathematical physics. In Partial differential equations and inverse problems, volume 362 of Contemp. Math., pages 241-276. Amer. Math. Soc., Providence, RI, 2004.

25. S. Z. Levendorskiı. Acoustic waves in perturbed periodic layer: a limiting absorption principle. Asymptot. Anal., 16(1):15-24, 1998.

26. K. Morgenröther and P. Werner. On the principles of limiting absorption and limit amplitude for a class of locally perturbed waveguides. I. Time-independent theory. Math. Methods Appl. Sci., 10(2):125-144, 1988.

27. E Mourre. Absence of singular continuous spectrum for certain self-adjoint operators. Communications in Mathematical Physics, 78(3):391-408, 1981.

28. Sergey A. Nazarov and Boris A. Plamenevsky. On radiation conditions for selfadjoint elliptic problems. Dokl. Akad. Nauk SSSR, 311 (3):532-536, 1990.

29. Sergey A. Nazarov and Boris A. Plamenevsky. Radiation principles for selfadjoint elliptic problems. Probl. Mat. Fiz., 13:192-244, 1991.

30. Sergey A. Nazarov and Boris A. Plamenevsky. Elliptic problems in domains with piecewise smooth boundaries, volume 13 of de Gruyter Expositions in Mathematics. Walter de Gruyter \& Co., Berlin, 1994.

31. Serguey A. Nazarov. The mandelshtam energy radiation conditions and the umovpoynting vector in elastic waveguides. Probl. Mat. anal., 72:101-146, 2013.

32. Serguey A. Nazarov. Umov-mandelshtam radiation conditions in elastic periodic waveguides. Sbornik: Mathematics, 205:7:953-982, 2014.

33. M. Notomi. Theory of light propagation in strongly modulated photonic crystals : Refractionlike behavior in the vicinity of the photonic bandgap. Phys. Rev. B, 62(16):10696-10705, 2000.

34. Josip Plemelj. Problems In the Sense of Riemann And Klein. New York: Interscience Publishers, 1964.

35. Maria Radosz. The principle of limit absorption and limit amplitude for periodic operators. Ph.D. thesis. Fakultät für Mathematik des KIT, Karlsruhe, France, 2009.

36. Michael Reed and Barry Simon. Methods of modern mathematical physics v. I-IV. Academic Press, New York, 1972-1978.

37. K. Sakoda. Optical Properties of Photonic Crystals. Springer Verlag Berlin, 2001.

38. Alexander V. Sobolev and Jonathan Walthoe. Absolute continuity in periodic waveguides. Proc. London Math. Soc. (3), 85(3):717-741, 2002.

39. Yulian-Karl Wasilievich Sokhotski. On definite integrals and functions used in series expansions. Doctor thesis, Saint Petersburg, 1873.

40. T. A. Suslina and R. G. Shterenberg. Absolute continuity of the spectrum of the magnetic schrödinger operator with a metric in a two-dimensional periodic waveguide. Algebra i Analiz, 14:159-206, 2002.

41. Ricardo Weder. Spectral and scattering theory for wave propagation in perturbed stratfied media. Springer Science and Business Media, 1990. 
42. Calvin H. Wilcox. Steady-state wave propagation in homogeneous anisotropic media. Arch. Rational Mech. Anal., 25(1967):201-242, 1967.

43. Calvin H Wilcox. Sound propagation in stratified fluids. Applied Mathematical Sciences, New York: Springer, 1984, 1, 1984. 